\title{
Supporting Information \\ Multistep Reaction Based De Novo Drug Design: Generating Synthetically Feasible Design Ideas
}

Brian B. Masek, * David S. Baker, Roman J. Dorfman, Karen DuBrucq, Victoria C. Francis, Stephan Nagy, Bree L. Richey, and Farhad Soltanshahi

Certara, 210 N. Tucker Blvd, Suite 350, Saint Louis, MO 63101 USA

*To whom correspondence should be addressed.E-mail: brian.masek@certara.com 


\section{Index of Reactions:}

\section{Heterocycle Formation - Nitrogen containing}

- $\quad$ Pictet-Spengler

- Benzimidazole via Condensation with Carboxylic_Acid/Ester

- Benzimidazole via Condensation with Aldehyde

- Niementowski Quinazoline Synthesis

- Terminal Tetrazole via Cycloaddition

- 2,5-Tetrazole via Cycloaddition

- $\quad$ 1,5-Tetrazole via Cycloaddition

- 1,4-Subst Cu Catalyzed Huisgen

- 1,5-Subst Ru Catalyzed Huisgen

- Huisgen with Disubstituted Alkyne

- 1,2,4-Triazole via Condensation with Acetohydrazide

- 1,2,4-Triazole via Condensation with Carboxylic Acid/Ester

- 3-Nitrile Pyridine via Condensation

- 1,3,5-Pyrazole via Condensation

- 2,4-Phthalazinone via Condensation

- Paal-Knorr Pyrrole

- Triaryl Imidazole via Condensation

- Fischer Indole Synthesis

- Friedlaender Quinoline

- Indole Synthesis

- Imidazole via Condensation

- Copper-Catalyzed Azide-Alkyne Cycloaddition

\section{Heterocycle Formation - Oxygen containing}

- $\quad$ Spiro Chromanone via Condensation

- Benzofuran via Cycloaddition

- Paal-Knorr Furan

- Pechmann Condensation

\section{Heterocycle Formation - Sulfur containing}

- Benzothiophene via Condensation

- Paal-Knorr Thiophene

\section{Heterocycle Formation - Multiple}

- Benzothiazole via Condensation

- Benzoxazole via Condensation with Aromatic Aldehyde

- Benzoxazole via Condensation with Carboxylic Acid

- Hantzsch Thiazole

- Oxadiazole via Condensation 
- Heterocycle Ring Formation

Heterocycle Formation - Dieckmann

- Dieckmann Condensation

\section{C-O Bond Formation}

- Williamson Ether

Arylation

- Biaryl via Decarboxylative Coupling

- Migita-Kosugi-Stille Coupling

- Chan-Lam Coupling

Heteroatom Alkylation and Arylation: $\mathrm{N}$-acylation to amide

- Reductive Amination

Heteroatom Alkylation and Arylation: Amide $\mathrm{N}$-alkylation

- Mitsunobu Imide

- Mitsunobu Sulfonamide

Heteroatom Alkylation and Arylation: O-substitution

- Mitsunobu Phenol

Heteroatom Alkylation and Arylation: Heteroaryl N-alkylation

- Mitsunobu Tetrazole

Heteroatom Alkylation and Arylation: N-arylation with Ar-X

- Buchwald-Hartwig

C-C Bond Formation: Suzuki Coupling

- $\underline{\text { Suzuki }}$

\section{C-C Bond Formation: Other}

- Piperidine Indole via Condensation

- Heteroatom Alkylation via Nucleophilic Attack

- Aldol Condensation 


\section{C-C Bond Formation: Sonogashira}

- $\underline{\text { Sonogashira }}$

\section{C-C Bond Formation: Heck}

- Mizoroki-Heck Terminal Vinyl

- Mizoroki-Heck Non-Terminal Vinyl

\section{C-C Bond Formation: Grignard}

- Grignard Carbonyl

- Grignard Alcohol

\section{C-C Bond Formation: Other Pd-Catalyzed Reactions}

- $\underline{\text { Negishi }}$

\section{C-C Bond Formation: Wittig Olefination}

- Wittig

\section{C-C Bond Formation: Functional Group Addition}

- Friedel-Crafts Alkylation

- Friedel-Crafts Acylation

- Michael-Addition

Acylation and Related Processes: $\mathbf{N}$-acylation to amide

- Schotten-Baumann Amide

Acylation and Related Processes: N-sulfonylation

- Sulfonamide

Amination

- Wöhler

- Thiourea via Nucleophilic Addition

- NHS Ester Reaction

- Heteroaromatic via Nucleophilic Substitution

- Ortho Substituted Nitroaniline via Nucleophilic Substitution

- Para Substituted Nitroaniline via Nucleophilic Substitution

Amide Formation 
- Weinreb-Nahm Amide

\section{Azide Reduction}

- $\quad$ Staudinger Reaction

\section{Oxime Rearrangement}

- $\quad$ Beckmann Rearrangement

\section{Reductions}

- $\quad$ Amide Reduction

- Imine Reduction

- Nitrile Reduction

- Ester Reduction

- Ketone Reduction

- Alkene Reduction

- Alkyne Reduction

- Nitroso Reduction

- Nitro to Hydroxylamine

- Nitro to Amine/Aniline

- Aliphatic Nitro to Oxime

- Aliphatic Nitro to Hydrocarbon

\section{Oxidation}

- $\quad$ Primary Alcohol [O] to Aldehyde

- Primary Alcohol [O] to Carboxylic Acid

- Secondary Alcohol [O] to Ketone

- Sulfide to Sulfoxide

- Sulfide to Sulfone

- Sulfoxide to Sulfone 


\section{Pictet-Spengler ${ }^{1,2,3,4,5,6,7}$}<smiles></smiles><smiles>[R]c1c([R])c([R])c2c(CCN)c[nH]c2c1[R]</smiles><smiles>[R]C=O</smiles>

\section{Reaction Notes:}

- This reaction can produce regioisomers for some reagents.

Return to Index of Reactions 


\section{Benzimidazole via Condensation with Carboxylic Acid/Ester ${ }^{1,8}$}

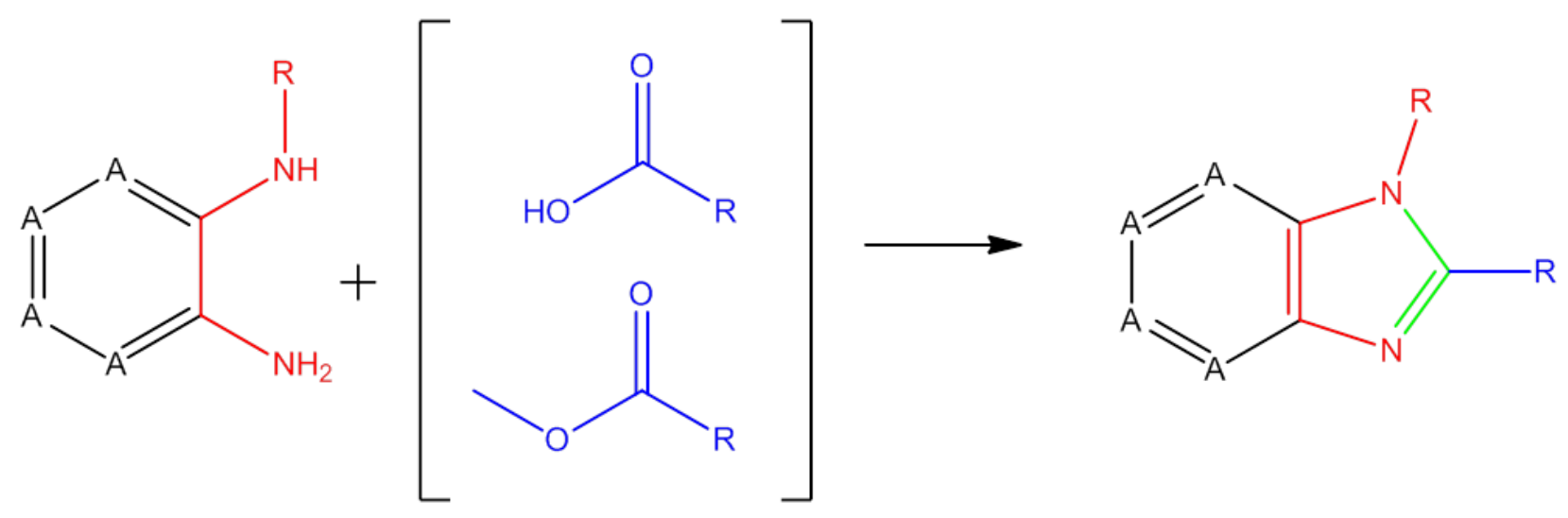

\section{Reaction Notes:}

- This reaction is possible with any six-membered aromatic heterocycle.

Return to $\underline{\text { Index of Reactions }}$ 


\section{Benzimidazole via Condensation with Aldehyde ${ }^{1,8,9,10}$}
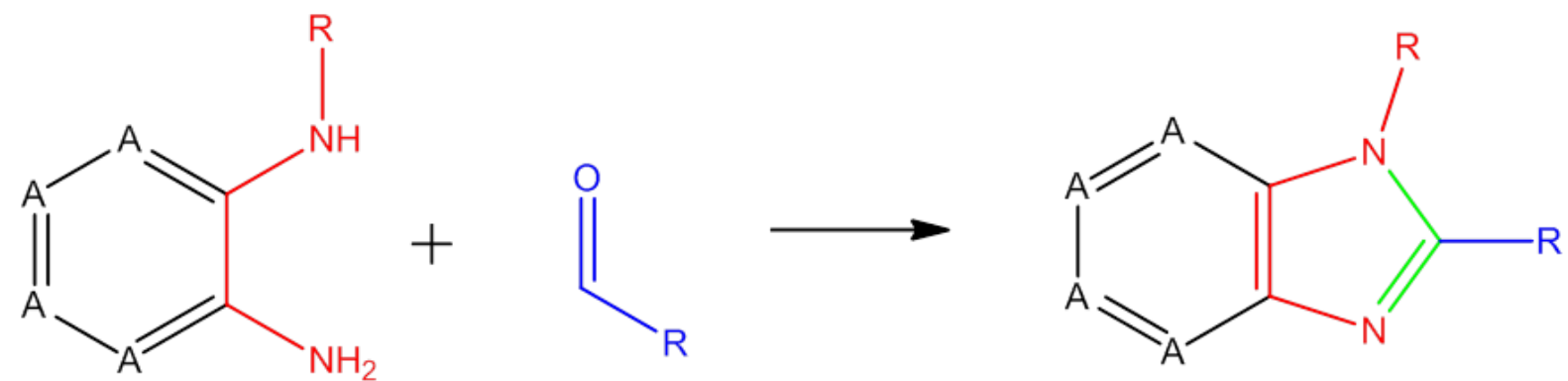

\section{Reaction Notes:}

- This reaction is possible with any six-membered aromatic heterocycle.

Return to Index of Reactions 
Niementowski Quinazoline Synthesis ${ }^{1,11}$
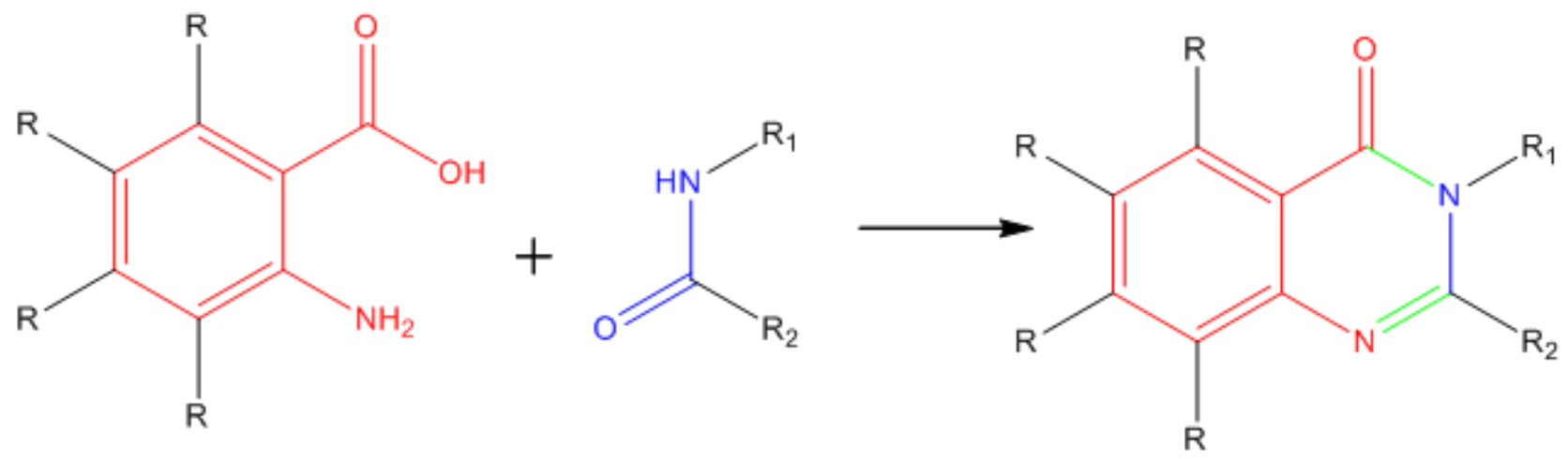

Return to Index of Reactions 


\section{Terminal Tetrazole via Cycloaddition ${ }^{1,12,13}$}
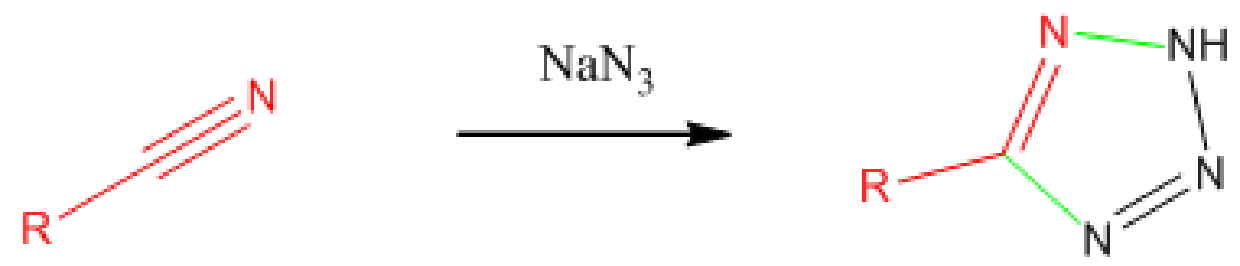

Return to Index of Reactions 


\section{2,5-Tetrazole via Cycloaddition ${ }^{1}$}
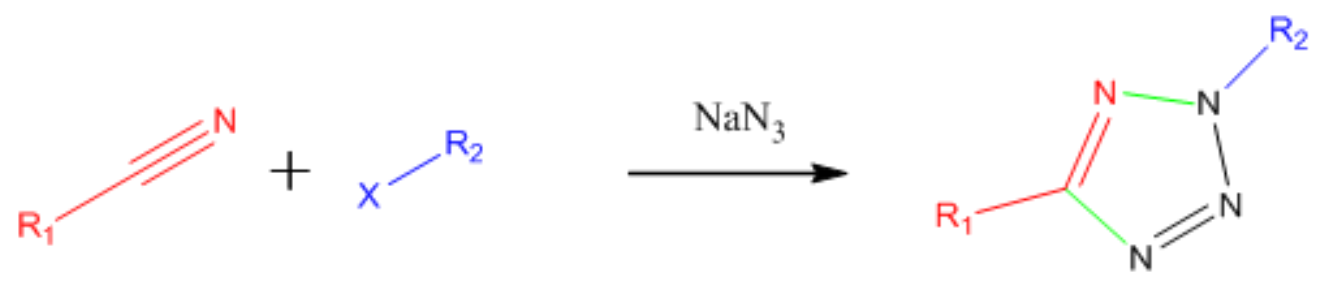

\section{Reaction Notes:}

- This reaction is not regioselective, but has an alternative product. There is an additional step where the halogen $(\mathrm{X})$ is substituted with azide $\left(\mathrm{NaN}_{3}\right)$.

Return to Index of Reactions 


\section{1,5-Tetrazole via Cycloaddition ${ }^{1}$}
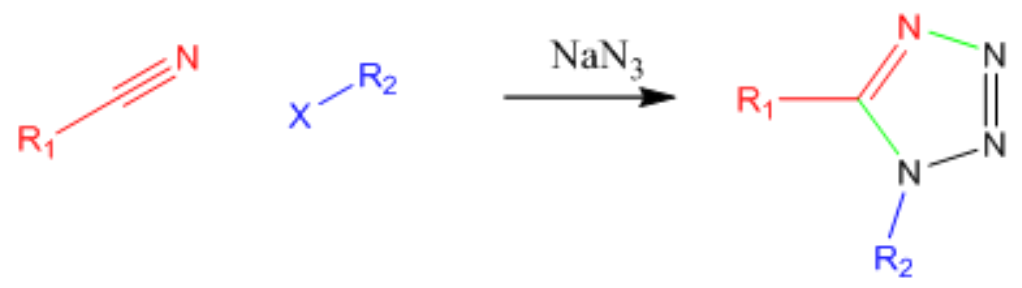

\section{Reaction Notes:}

- This reaction is not regioselective, but has an alternative product. There is an additional step where the halogen $(\mathrm{X})$ is substituted with azide $\left(\mathrm{NaN}_{3}\right)$.

Return to Index of Reactions 


\section{1,4-Subst Cu Catalyzed Huisgen ${ }^{1,14,15}$}

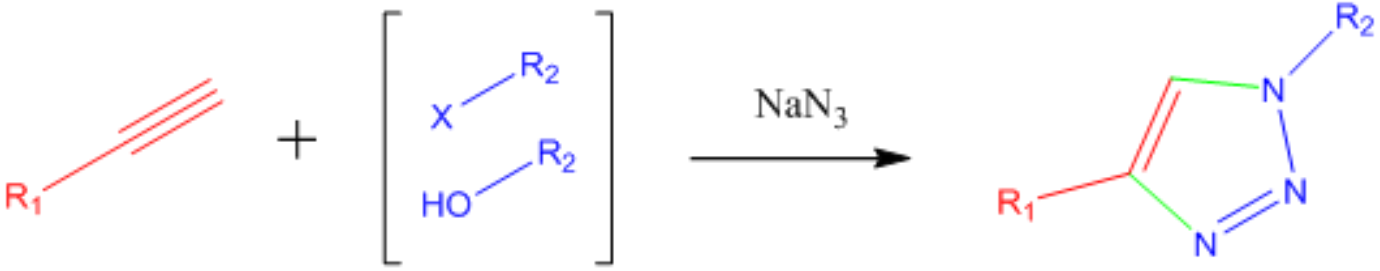

\section{Reaction Notes:}

- $\mathrm{X}=\mathrm{Cl}, \mathrm{Br}, \mathrm{I}$

- $\quad \mathrm{R} 1=$ aryl, alkyl

- $\quad$ R2 = aliphatic carbon

- The halogen or hydroxyl group is substituted under Mitsunobu conditions with $\mathrm{NaN}_{3}$. The stereochemistry must be considered at the secondary halides/alcohols.

Return to Index of Reactions 


\section{1,5-Subst Ru Catalyzed Huisgen ${ }^{1,14}$}

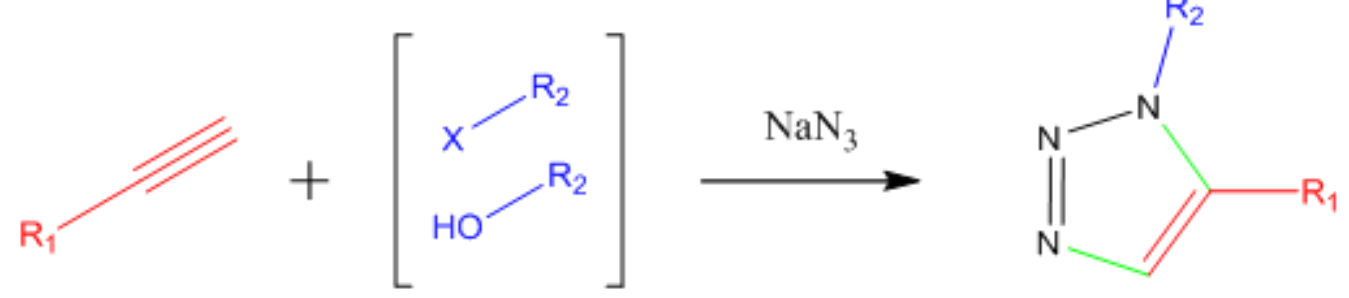

\section{Reaction Notes:}

- $\mathrm{X}=\mathrm{Cl}, \mathrm{Br}, \mathrm{I}$

- $\mathrm{R} 1=$ aryl, alkyl

- $\mathrm{R} 2$ = aliphatic carbon

- The halogen or hydroxyl group is substituted under Mitsunobu conditions with $\mathrm{NaN}_{3}$. The stereochemistry must be considered at the secondary halides/alcohols.

Return to Index of Reactions 


\section{Huisgen with Disubstituted Alkyne $e^{1,14}$}

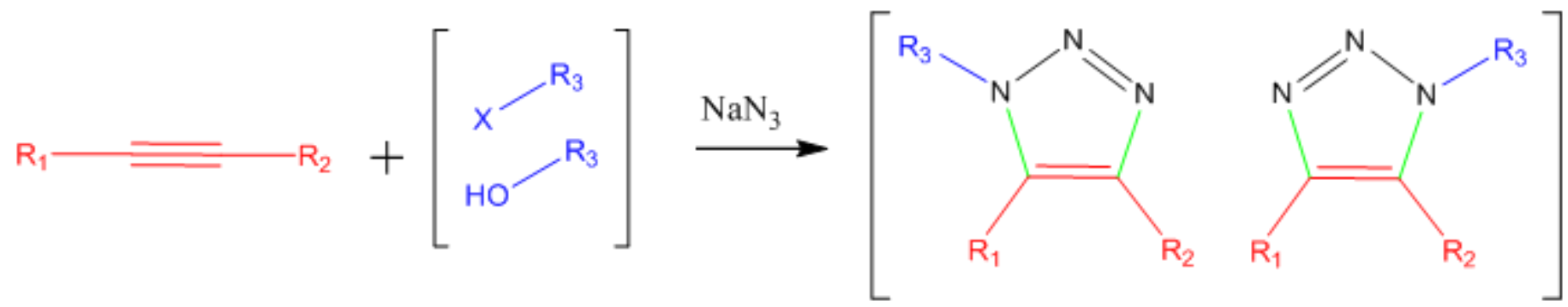

\section{Reaction Notes:}

- $\mathrm{X}=\mathrm{Cl}, \mathrm{Br}, \mathrm{I}$

- $\mathrm{R} 1=$ aryl, alkyl

- $\mathrm{R} 2$ = aliphatic carbon

- This reaction is not regioselective rather asymmetrically substituted alkynes form both regioisomers. The halogen or hydroxyl group is substituted under Mitsunobu conditions with $\mathrm{NaN}_{3}$. The stereochemistry must be considered at the secondary halides/alcohols.

Return to Index of Reactions 


\section{1,2,4-Triazole via Condensation with Acetohydrazide ${ }^{1}$}

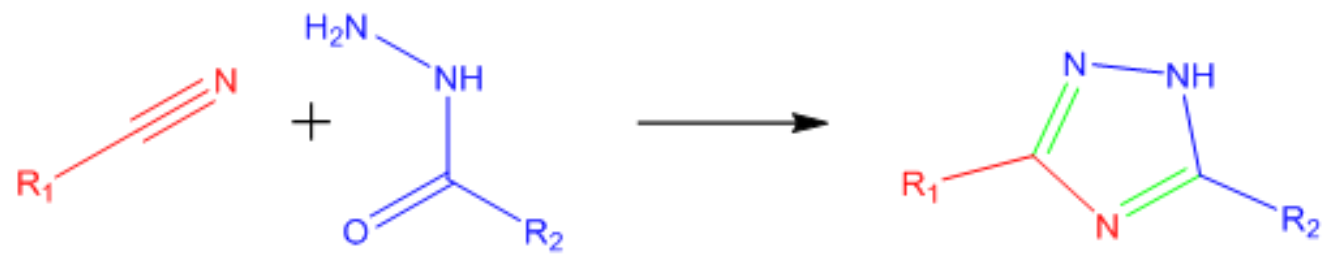

Return to Index of Reactions 


\section{1,2,4-Triazole via Condensation with Carboxylic Acid/Ester ${ }^{\text {Error! Reference source not found. }}$}
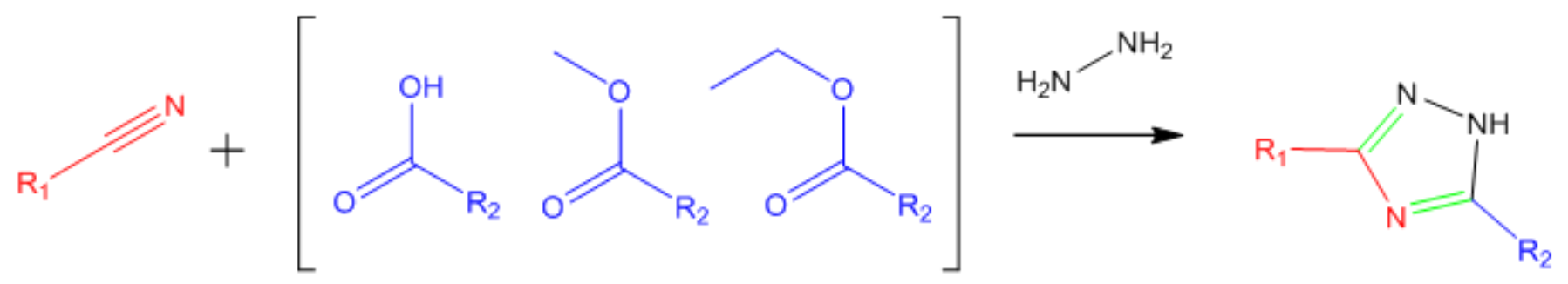

\section{Reaction Notes:}

- There is an additional nucleophilic substitution with hydrazine step.

Return to Index of Reactions 


\section{3-Nitrile Pyridine via Condensation ${ }^{1}$}
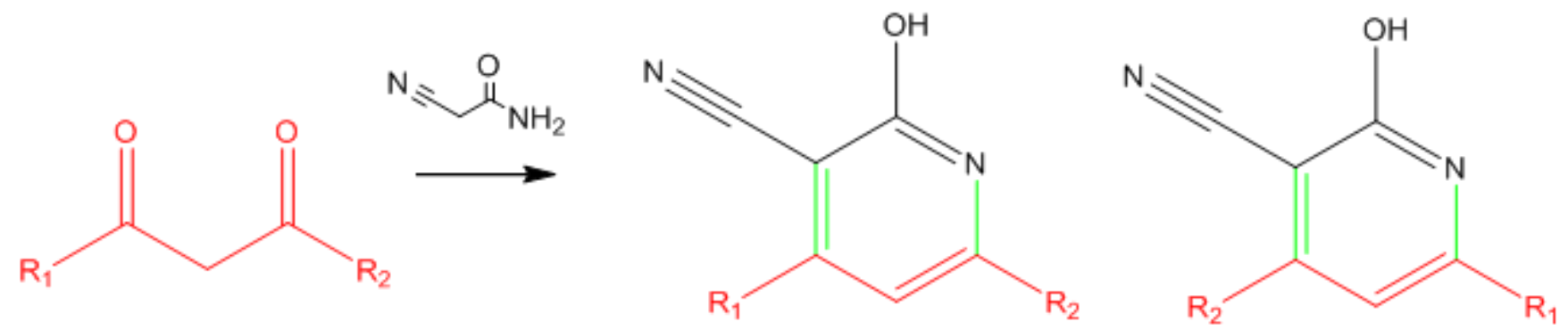

\section{Reaction Notes:}

- R1 and R2 must be an aromatic or aliphatic carbon and not carbonyl/carboxylic acid.

- Central carbon of reactant must have at least one hydrogen atom attached.

- Only one of the carbonyl groups of the reactant can be part of the product ring.

- This reaction has the potential to produce regioisomers due to symmetric substructure definition.

Return to Index of Reactions 


\section{1,3,5-Pyrazole via Condensation ${ }^{1}$}

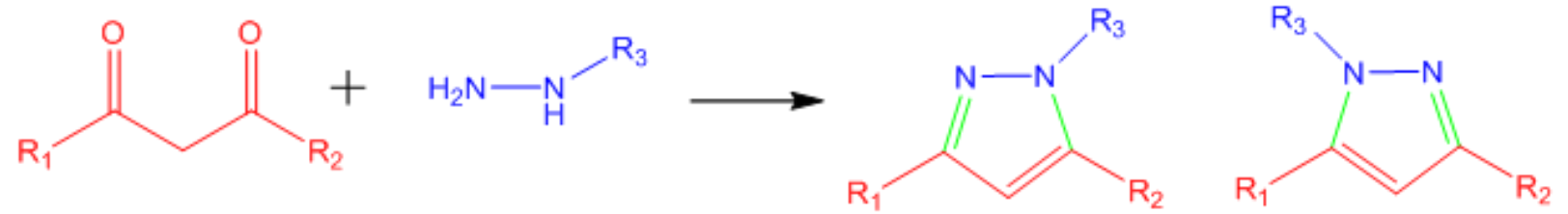

\section{Reaction Notes:}

- $\quad \mathrm{R} 1$ and R2 must be an aromatic or aliphatic carbon and not carbonyl/carboxylic acid.

- $\mathrm{R} 3=\mathrm{H}, \mathrm{C}$, or possibly even $\mathrm{C}=\mathrm{O}$

- Central carbon of reactant must have at least one hydrogen atom attached.

- Only one of the carbonyl groups of the reactant can be part of the product ring.

- This reaction has the potential to produce regioisomers due to symmetric substructure definition.

Return to Index of Reactions 


\section{2,4-Phthalazinone via Condensation ${ }^{1}$}
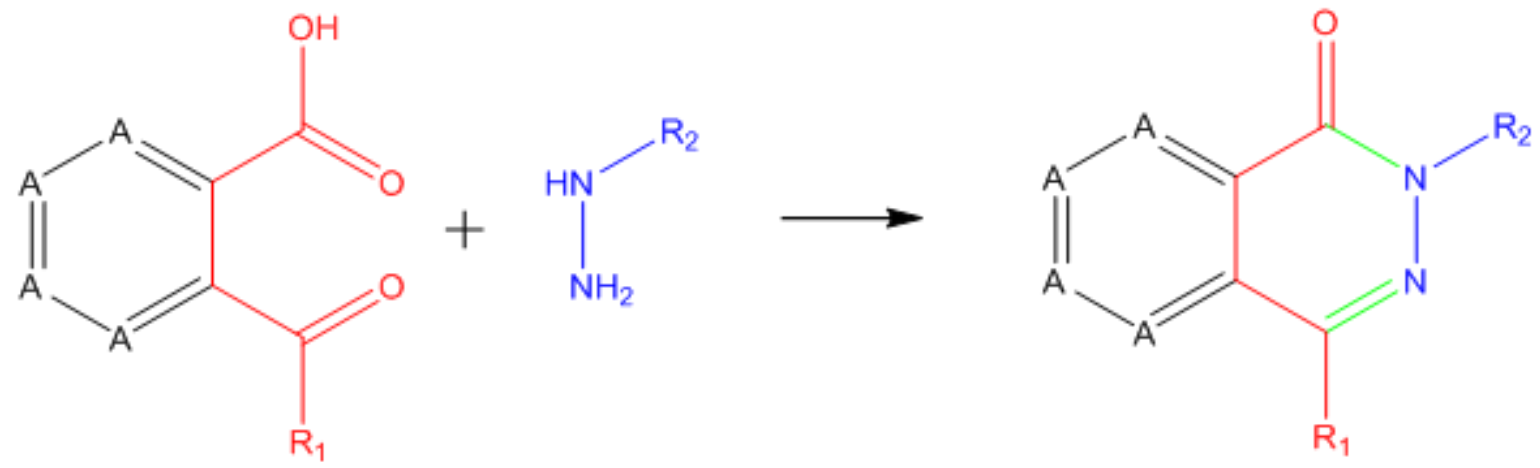

\section{Reaction Notes:}

- $\mathrm{R} 2$ must be $\mathrm{C}$, but not a $\mathrm{C}=\mathrm{O}, \mathrm{C}=\mathrm{S}$, or $\mathrm{C}=\mathrm{N}$

- This reaction is possible with any six-membered aromatic heterocycle, even if it is substituted.

Return to Index of Reactions 


\section{Paal-Knorr $^{1,16,17,18}$}

\section{Pyrrole}

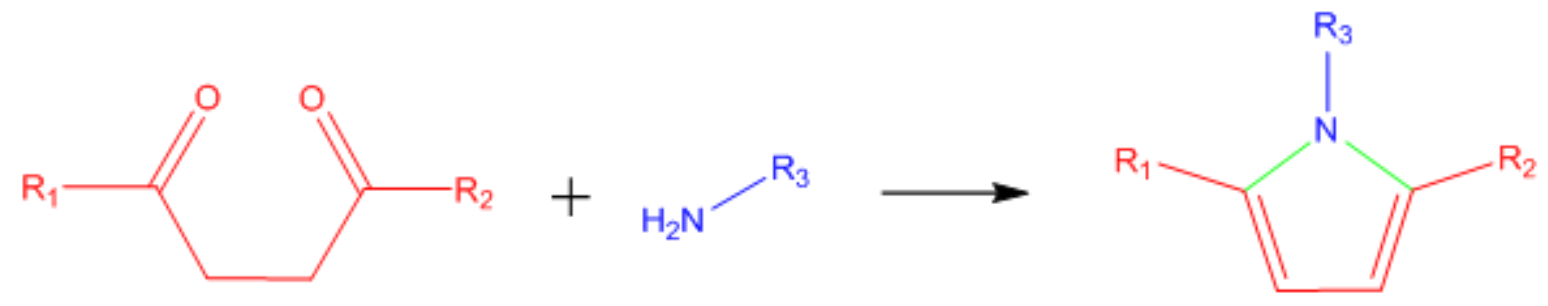

\section{Reaction Notes:}

- The two central carbons of first reactant may be substituted, but must contain at least one hydrogen atom.

- The second reactant must be a primary amine.

\section{Furan}
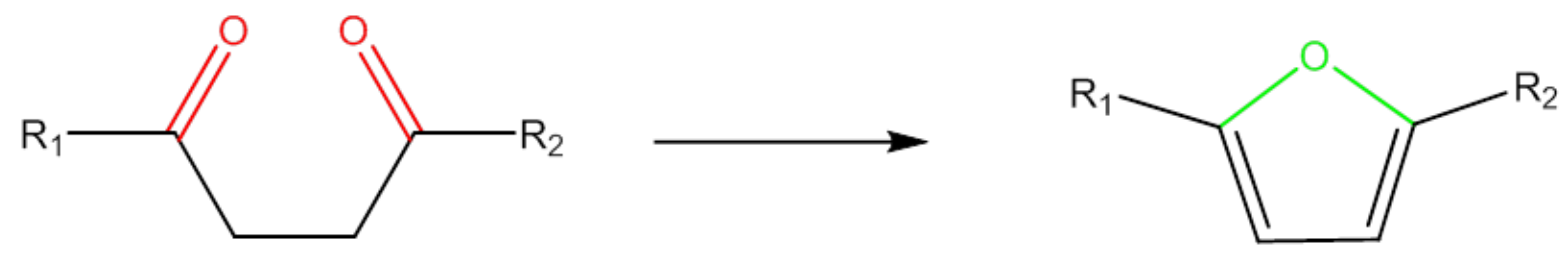

\section{Reaction Notes:}

- Acid-catalyzed synthesis results in hemiacetal intermediate followed by furan formation. 


\section{Thiophene}

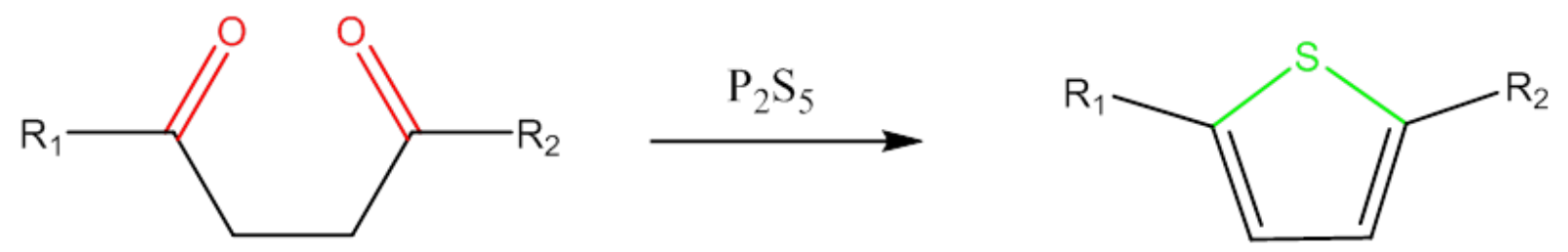

\section{Reaction Notes:}

- Addition of sulfonization agent $\mathrm{P}_{2} \mathrm{~S}_{5}$ is required to produce intermediate thioketone.

- Acid-catalyzed reaction results in thiophene.

Return to Index of Reactions 


\section{Triaryl Imidazole via Condensation ${ }^{1}$}
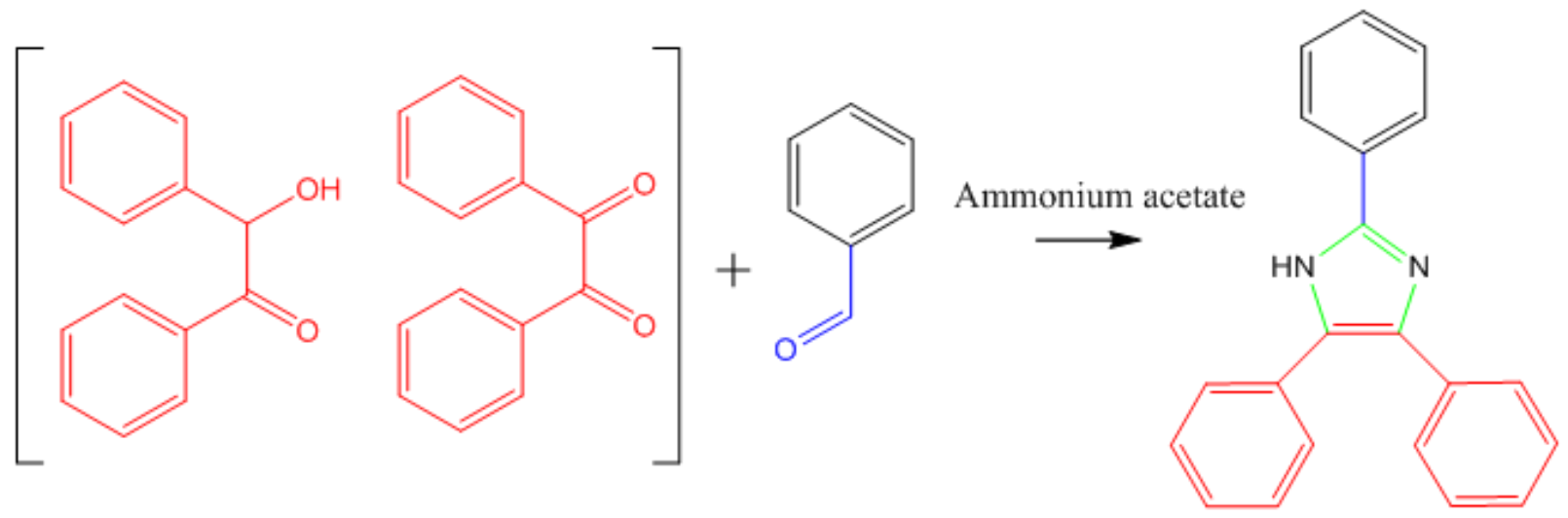

\section{Reaction Notes:}

- The first reactant may be a keto or a hydroxyl group.

- The second reactant must be an aldehyde connected to any aromatic system.

Return to Index of Reactions 


\section{Fischer Indole Synthesis ${ }^{1}$}<smiles></smiles>

\section{Reaction Notes:}

- This reaction can produce regioisomers for some reagents.

Return to Index of Reactions 


\section{Friedlaender Quinoline ${ }^{1,19}$}<smiles>[R]c1c([R])c([R])c(C=O)c(N)c1[R]</smiles>

Return to Index of Reactions

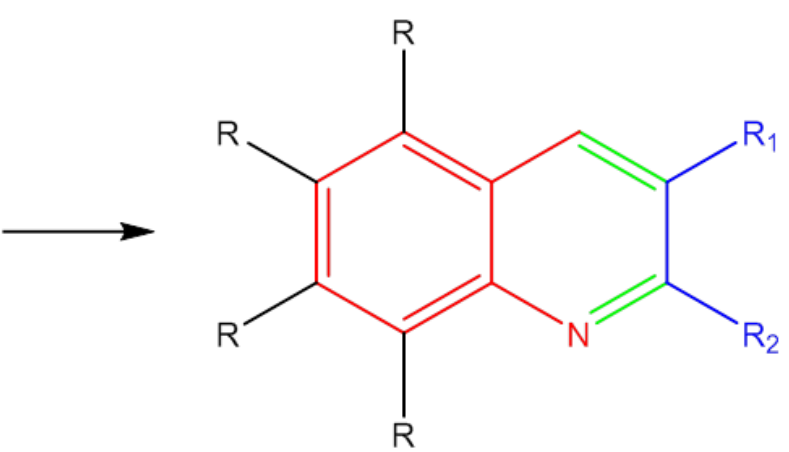




\section{Indole Synthesis ${ }^{1,20}$}
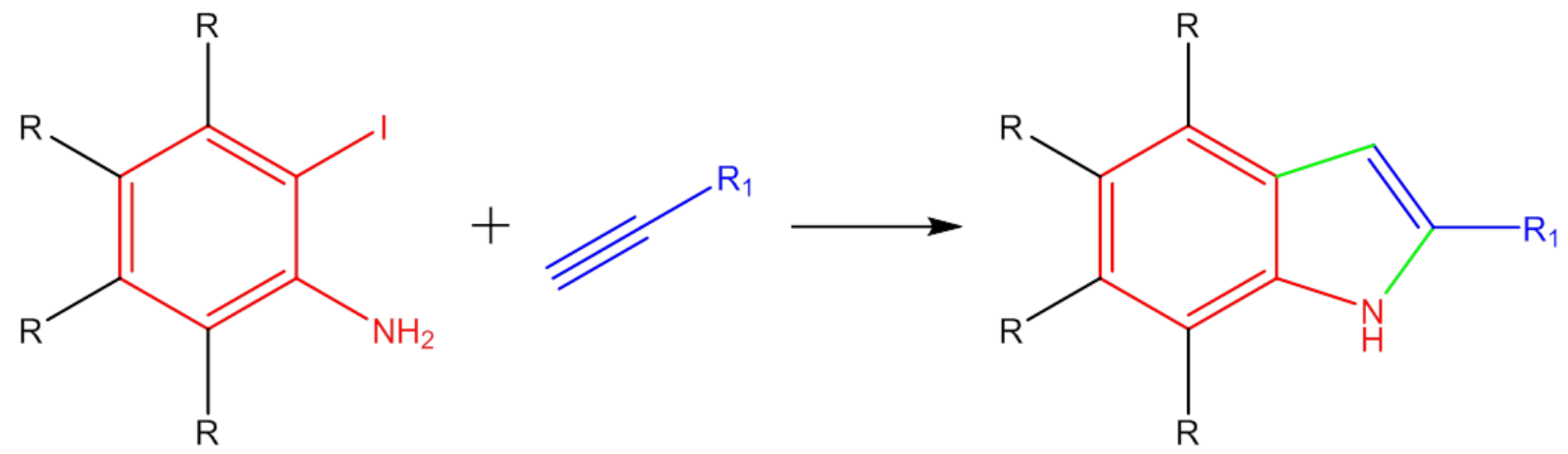

\section{Reaction Notes:}

- Br or I allowed on the heterocyclic reactant.

Return to Index of Reactions 


\section{Imidazole via Condensation $^{1}$}

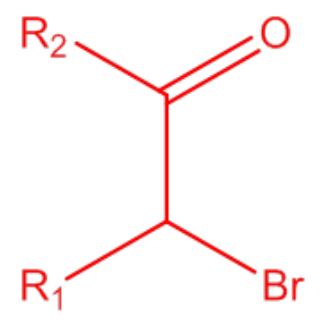

$+$

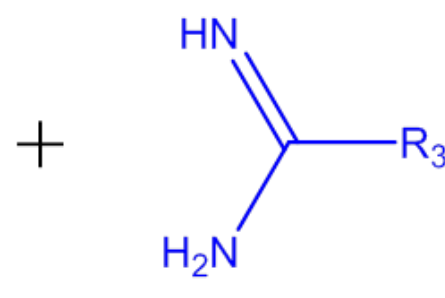

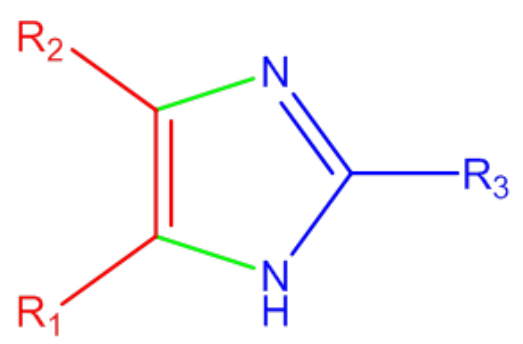

\section{Reaction Notes:}

- R1 and R2: The first atom must be a C

- R3: aryl, N

Return to Index of Reactions 


\section{Copper-Catalyzed Azide-Alkyne Cycloaddition ${ }^{21,22}$}

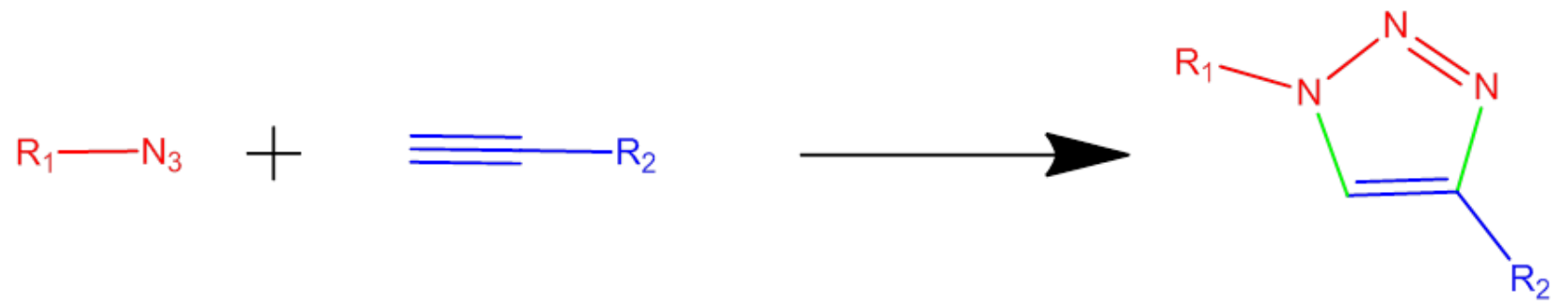

\section{Reaction Notes:}

- R1: Alkyl or $\mathrm{CH}_{2} \mathrm{OBn}$

- R2: $\mathrm{Ph}$ or $\mathrm{CO}_{2} \mathrm{H}$

Return to Index of Reactions 


\section{Spiro Chromanone via Condensation ${ }^{1}$}<smiles>[R]c1c([R])c(O)c(C(C)C)c(C(C)C)c1[R]</smiles><smiles>[R]c1c([R])c([R])c2c(c1[R])OC1(CCN([R])CC1)CC2=O</smiles>

Return to Index of Reactions 


\section{Benzofuran via Cycloaddition ${ }^{1}$}

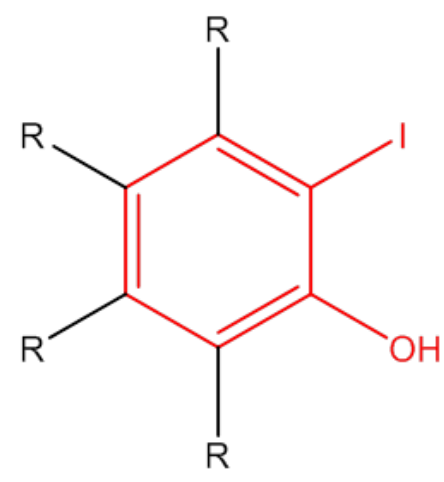

\section{Reaction Notes:}

- $\mathrm{Br}$ or I are allowed on the heterocyclic reactant.

Return to Index of Reactions

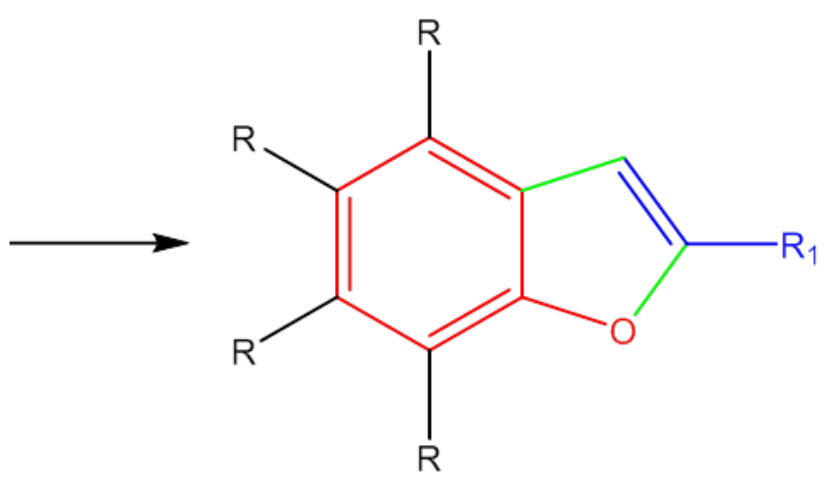




\section{Pechmann Condensation ${ }^{23}$}
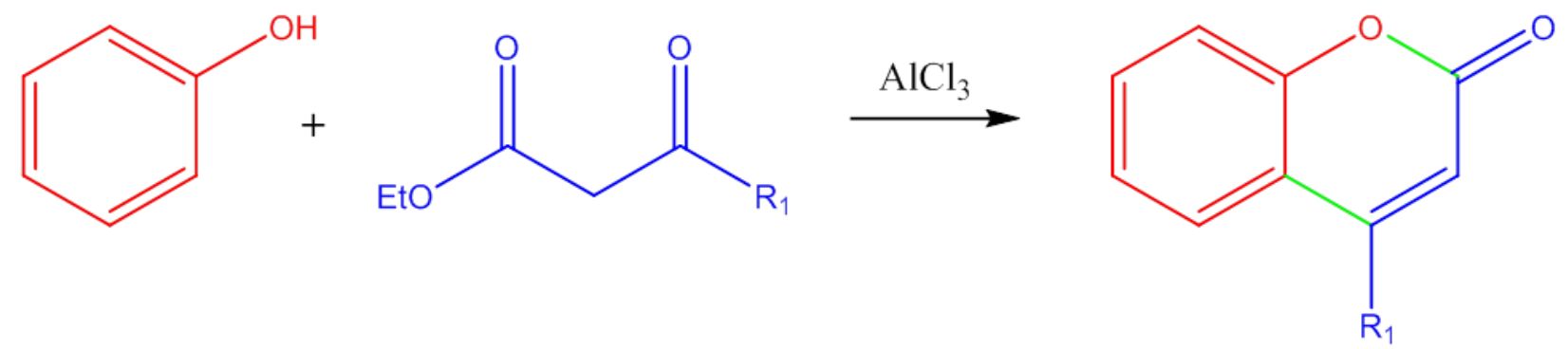

\section{Reaction Notes:}

- Requires addition of Lewis Acid $\mathrm{AlCl}_{3}$.

Return to Index of Reactions 


\section{Benzothiophene via Condensation ${ }^{1}$}<smiles>[R]c1c([R])c([R])c(SC)c(I)c1[R]</smiles>
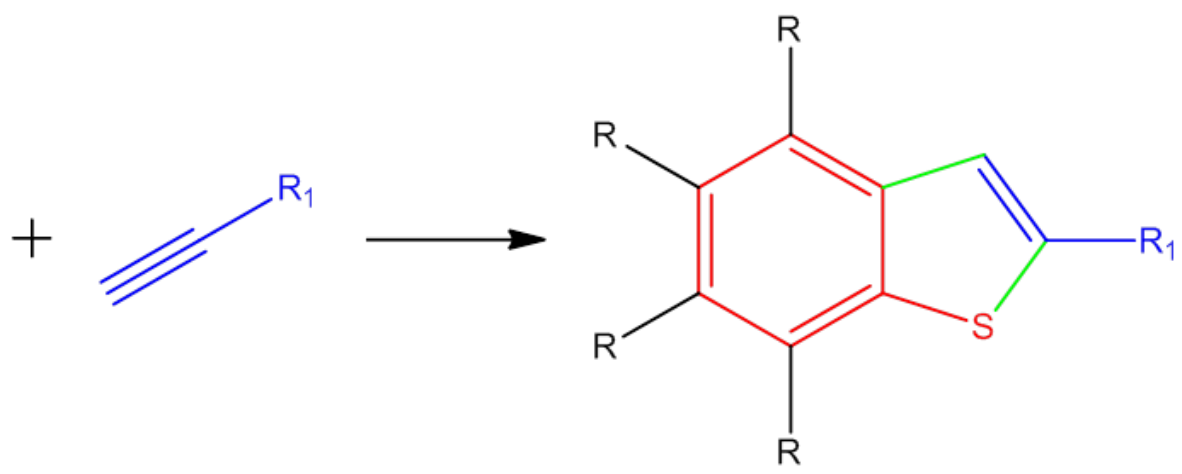

\section{Reaction Notes:}

- $\mathrm{Br}$ or I are allowed on the heterocyclic reactant.

Return to Index of Reactions 


\section{Benzothiazole via Condensation $^{1}$}
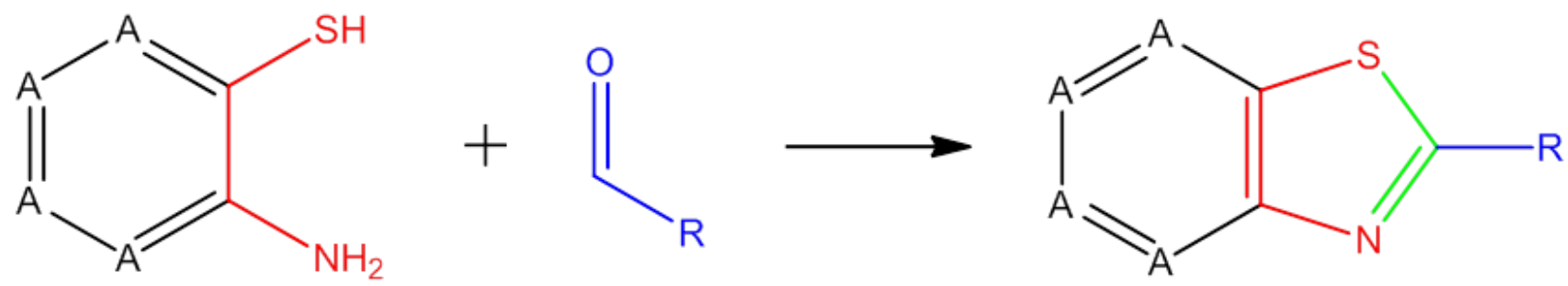

\section{Reaction Notes:}

- This reaction is possible with any six-membered heterocycle.

Return to Index of Reactions 


\section{Benzoxazole via Condensation with Aromatic Aldehyde ${ }^{1}$}
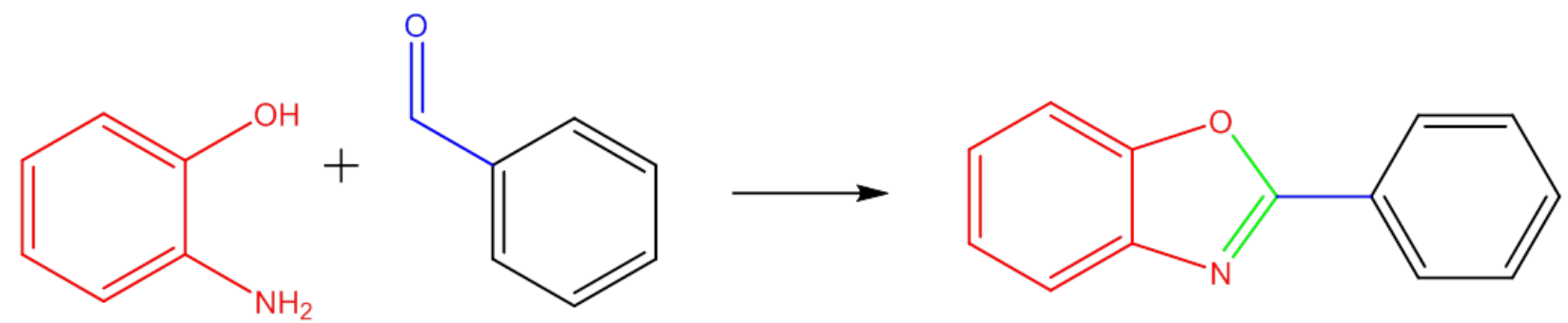

Return to Index of Reactions 


\section{Benzoxazole via Condensation with Carboxylic Acid ${ }^{1}$}
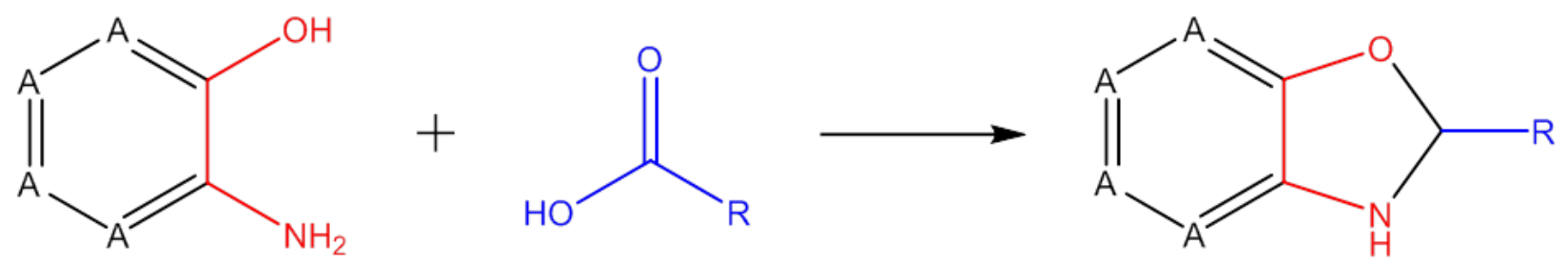

\section{Reaction Notes:}

- This reaction is possible with any six-membered heterocycle.

Return to Index of Reactions 


\section{Hantzsch Thiazole ${ }^{1}$}
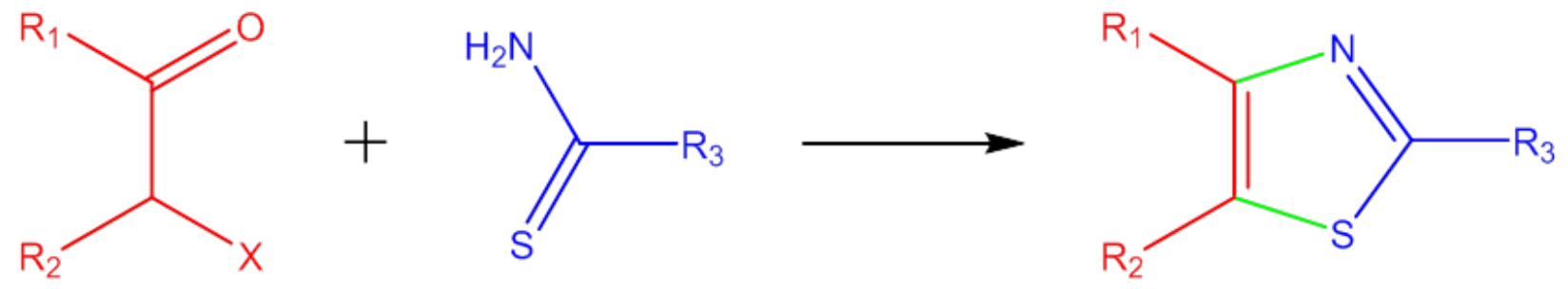

\section{Reaction Notes:}

- $\mathrm{X}: \mathrm{Cl}, \mathrm{Br}$, or I

- Neither carbons in the $\mathrm{C}=\mathrm{O}$ or $\mathrm{C}-\mathrm{X}$ groups cannot be part of a ring

Return to Index of Reactions 


\section{Oxadiazole via Condensation $^{1}$}
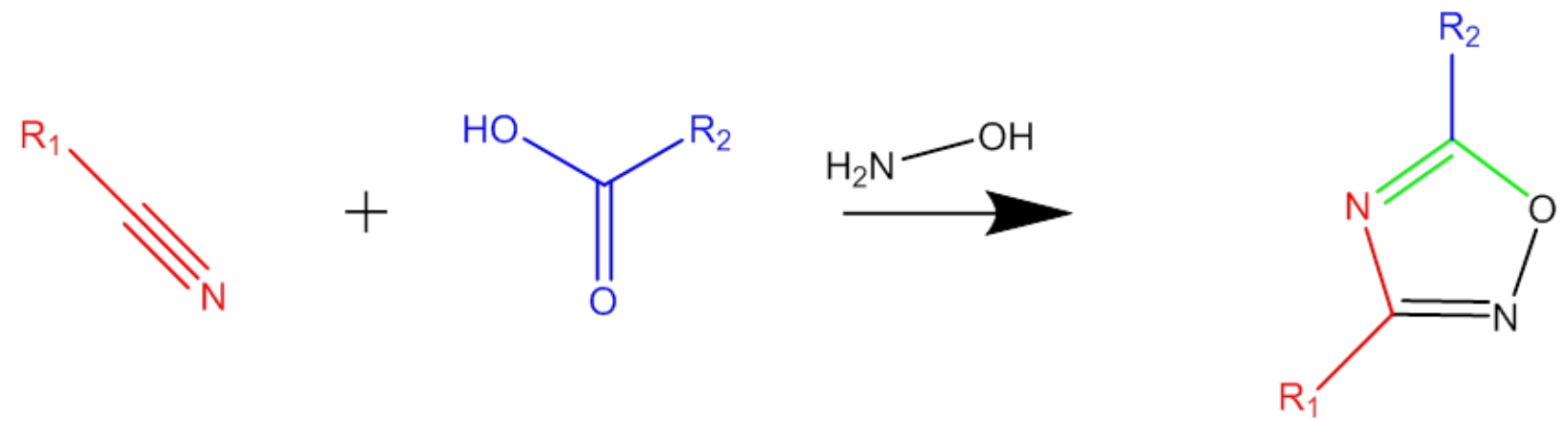

\section{Reaction Notes:}

- The nitrile must be converted to an amidoxime by the hydroxylamine reagent prior to the reaction.

Return to Index of Reactions 


\section{Heterocycle Ring Formation ${ }^{24}$}
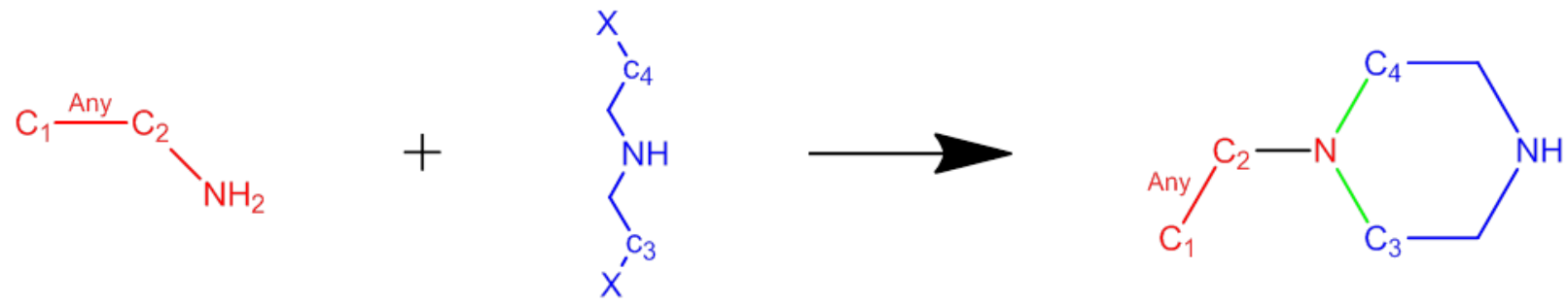

\section{Reaction Notes:}

- $\quad$ C1 should not have a $=\mathrm{O},=\mathrm{S}$, or a halogen attached.

- $\quad \mathrm{C} 2$ should not have $\mathrm{a}=\mathrm{O},=\mathrm{S}$, or $=\mathrm{N}$ attached.

- C3 \& C4 should have 2 hydrogens attached.

- The other carbon and nitrogen in the second reagent can be substituted and be a C, N, O, or $\mathrm{S}$.

- $\mathrm{X}$ can be a $\mathrm{Cl}$ or $\mathrm{Br}$.

Return to Index of Reactions 


\section{Dieckmann Condensation ${ }^{25,26,27}$}

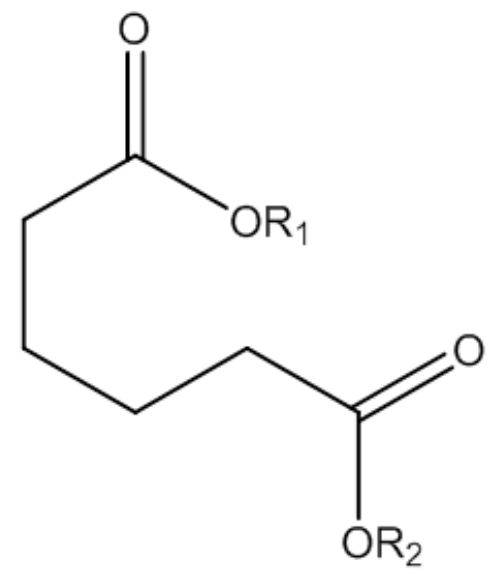

\section{Reaction Notes:}

- Intramolecular condensation reaction.

- R groups can be alkyl or aryl.

- Addition of alkoxide base necessary to drive reaction.

Return to Index of Reactions

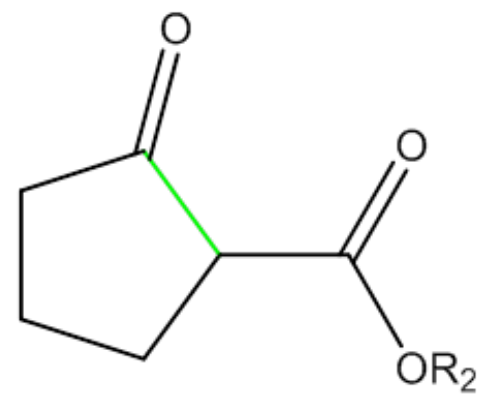

$\mathrm{OR}_{2}$ 


\section{Williamson Ether ${ }^{1}$}
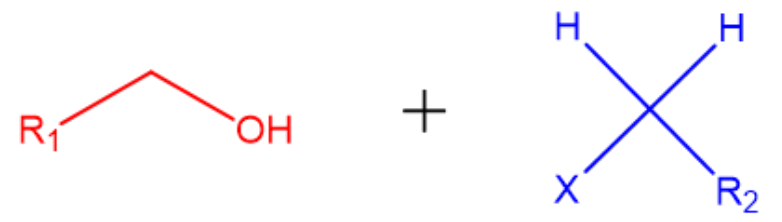

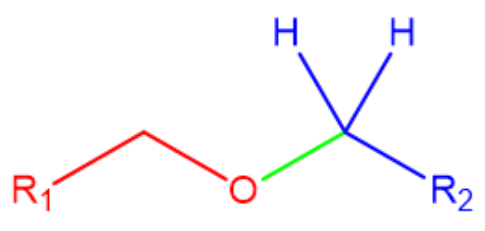

\section{Reaction Notes:}

- $\quad \mathrm{X}$ : Must be a $\mathrm{Cl}, \mathrm{Br}$, or I primary alkyl halide.

- The hydroxy group may be attached to an aromatic or aliphatic $\left(1^{0}, 2^{0}\right.$, or $\left.3^{0}\right)$ carbon.

Return to Index of Reactions 


\section{Biaryl via Decarboxylative Coupling ${ }^{1,28}$}
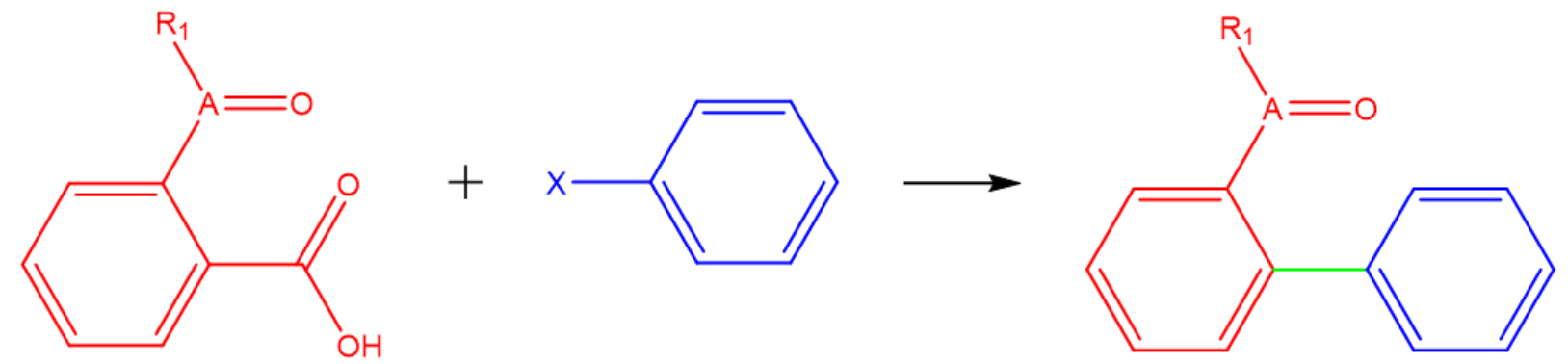

\section{Reaction Notes:}

- A: C, S, or N

- $\mathrm{X}: \mathrm{Cl}, \mathrm{Br}$, or I

Return to Index of Reactions 


\section{Migita-Kosugi-Stille Coupling ${ }^{1,29}$}

$\mathrm{R}_{1}-\mathrm{X}_{1}+\mathrm{X}_{2}-\mathrm{Ar}$<smiles>[R7][Al]</smiles>

\section{Reaction Notes:}

- Reactant one must be transformed into organotin (stannane) first.

- R1: vinyl $(\mathrm{C}=\mathrm{C})$, aryl

- X1: Br, I

- Ar: any aromatic system

- $\mathrm{X} 2: \mathrm{Cl}, \mathrm{Br}, \mathrm{I}$

Return to Index of Reactions 


\section{Chan-Lam Coupling ${ }^{1,30}$}
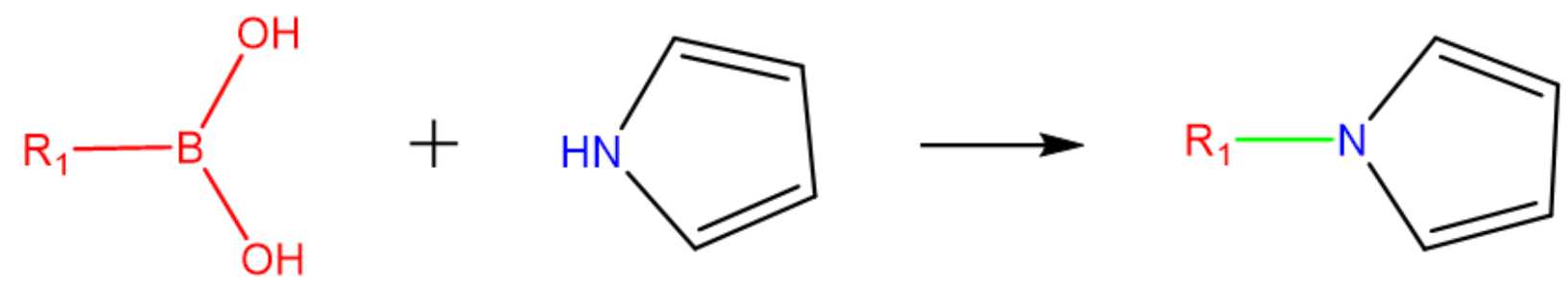

\section{Reaction Notes:}

- This reaction works with various 5-membered aromatic rings that contain NH even if they are fused to other rings.

- Regioisomers are likely to be produced in the case where protomers of the 5-membered heterocycle exist.

- R1: aryl

Return to Index of Reactions 


\section{Reductive Amination ${ }^{1}$}

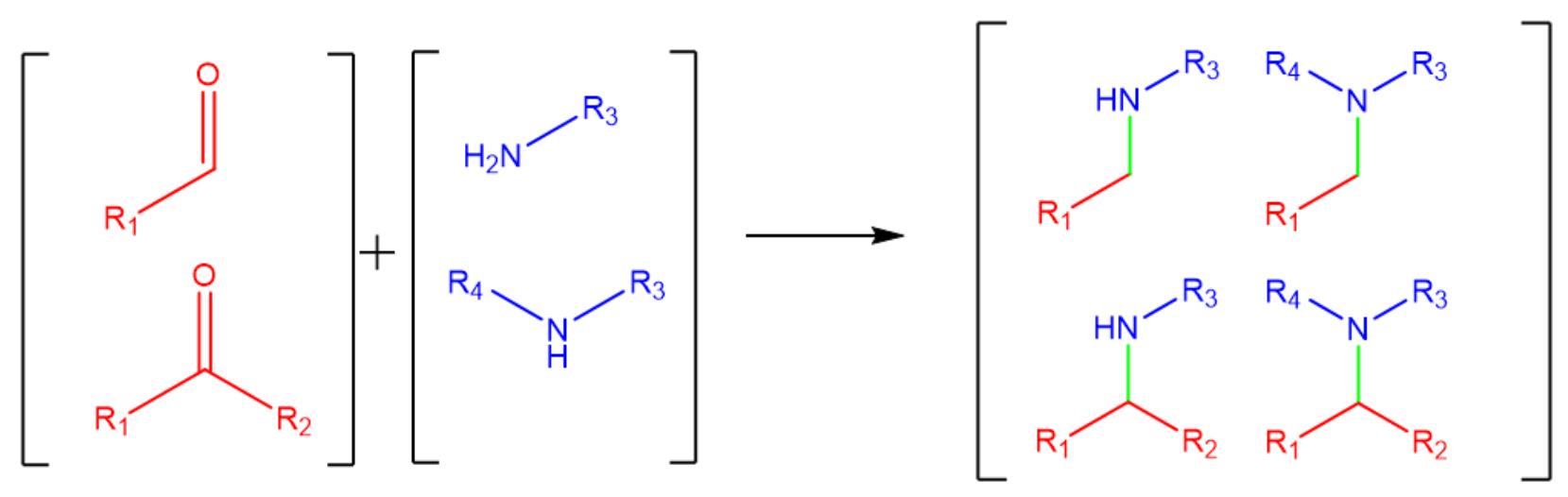

Return to Index of Reactions 


\section{Mitsunobu Imide ${ }^{1}$}

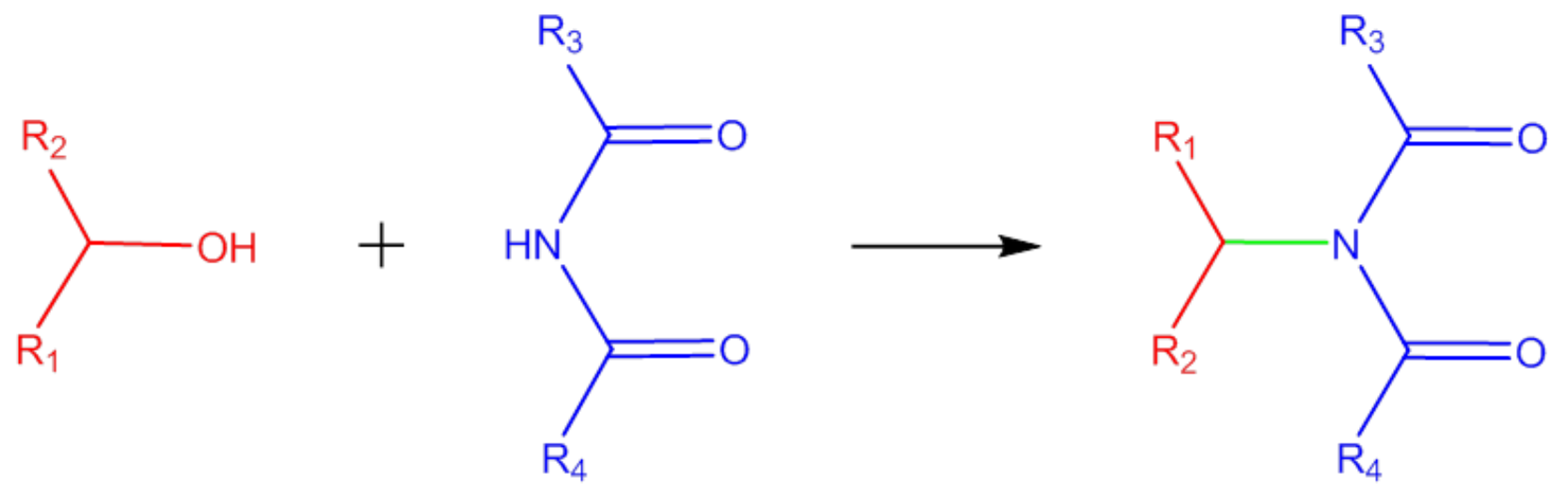

\section{Reaction Notes:}

- This reaction causes an inversion of stereochemistry at chiral centers.

- There are no restrictions on R1, R3, and R4; however, R2 must be either hydrogen or carbon.

Return to Index of Reactions 


\section{Mitsunobu Sulfonamide ${ }^{1}$}

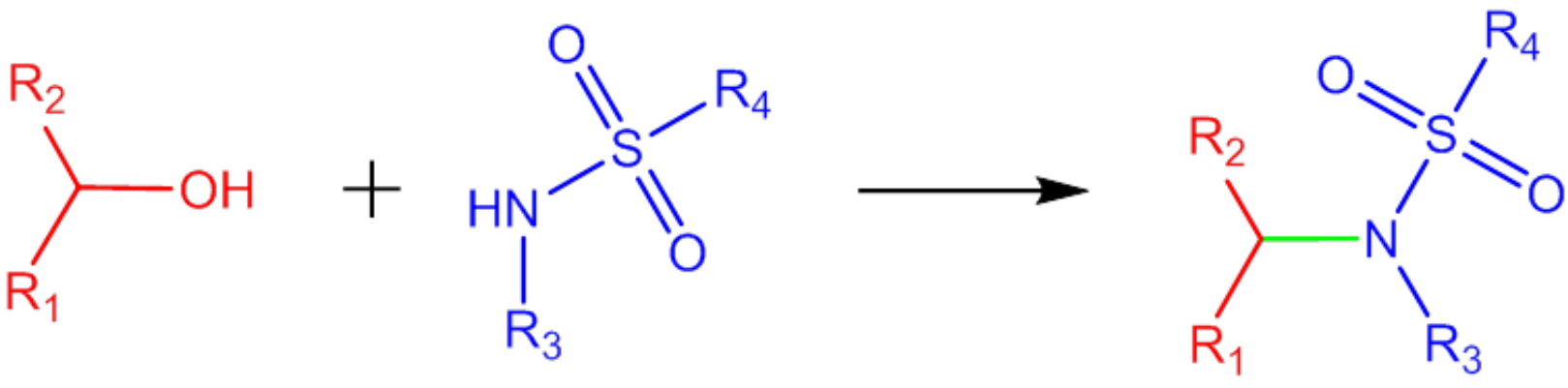

\section{Reaction Notes:}

- This reaction causes an inversion of stereochemistry at chiral centers.

- There are no restrictions on R1, R3, and R4; however, R2 must be either hydrogen or carbon.

Return to Index of Reactions 


\section{Mitsunobu Phenol ${ }^{1}$}

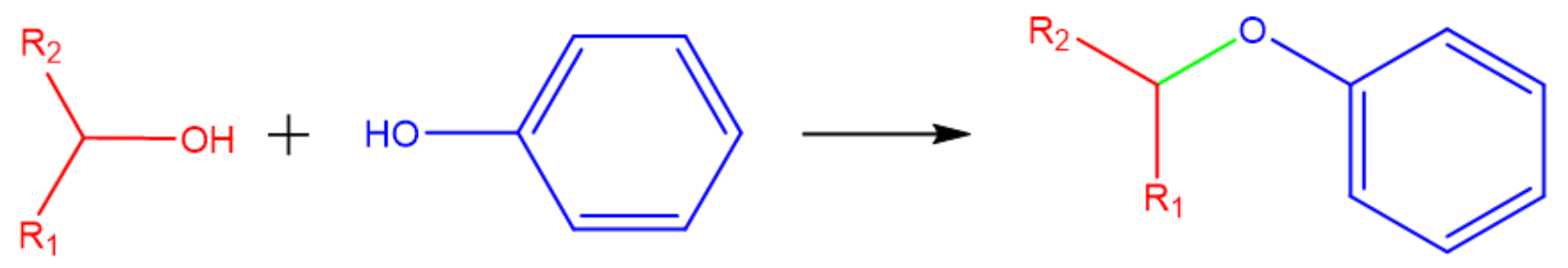

\section{Reaction Notes:}

- This reaction causes an inversion of stereochemistry at chiral centers.

- There are no restrictions on R1; however, R2 must be either hydrogen or carbon.

Return to Index of Reactions 


\section{Mitsunobu Tetrazole ${ }^{1}$}

Mitsunobu 1,5-Tetrazole (tautomers)<smiles>[R]C([R])O</smiles><smiles>[R3]c1nnn[nH]1</smiles>

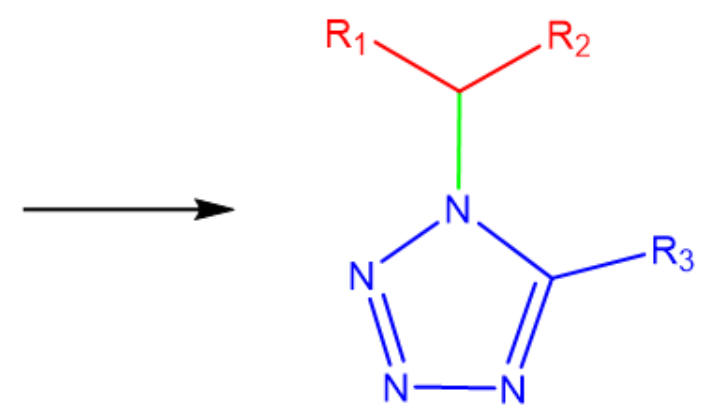<smiles>[R]C([R])O</smiles><smiles>[R3]c1nn[nH]n1</smiles><smiles>[R3]c1nnnn1C([R2])[R]</smiles>

Mitsunobu 2,5-Tetrazole (tautomers)<smiles>[R]C([R])O[I-]</smiles><smiles>[R]c1nnn[nH]1</smiles><smiles>CCCC</smiles><smiles>[R]C([R])O</smiles><smiles>[R3]c1nn[nH]n1</smiles><smiles>[R3]c1nnn(C([R])[R])n1</smiles> 


\section{Reaction Notes:}

- This reaction causes an inversion of stereochemistry at chiral centers.

- This reaction is not regioselective. An alternative product is substituted at the $\mathrm{N}$ at position 2 instead of 1 .

- There are no restrictions on R1 or R3; however, R2 must be either hydrogen or carbon.

Return to $\underline{\text { Index of Reactions }}$ 


\section{Buchwald-Hartwig ${ }^{1}$}

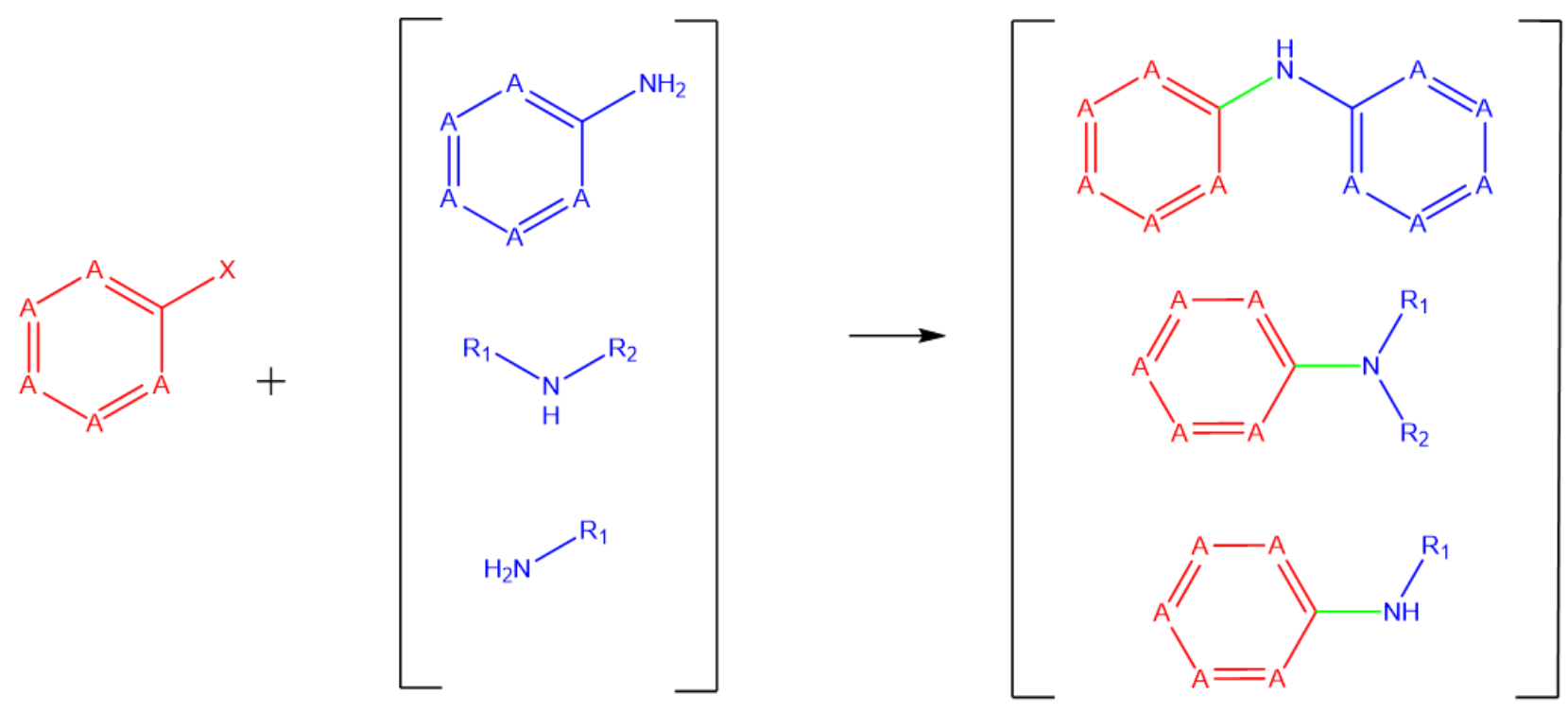

\section{Reaction Notes:}

- $\mathrm{X}: \mathrm{Cl}, \mathrm{Br}, \mathrm{I}$

- A: N,C

Return to Index of Reactions 


\section{Suzuki $^{1}$}

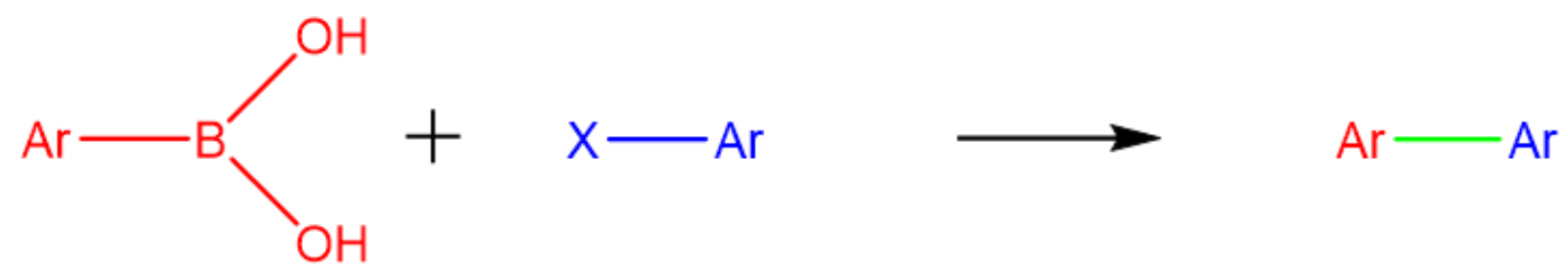

\section{Reaction Notes:}

- Any boronic acid (including cyclic structures)

- $\mathrm{X}: \mathrm{Cl}, \mathrm{Br}, \mathrm{I}$

Return to Index of Reactions 


\section{Piperidine Indole via Condensation ${ }^{1}$}

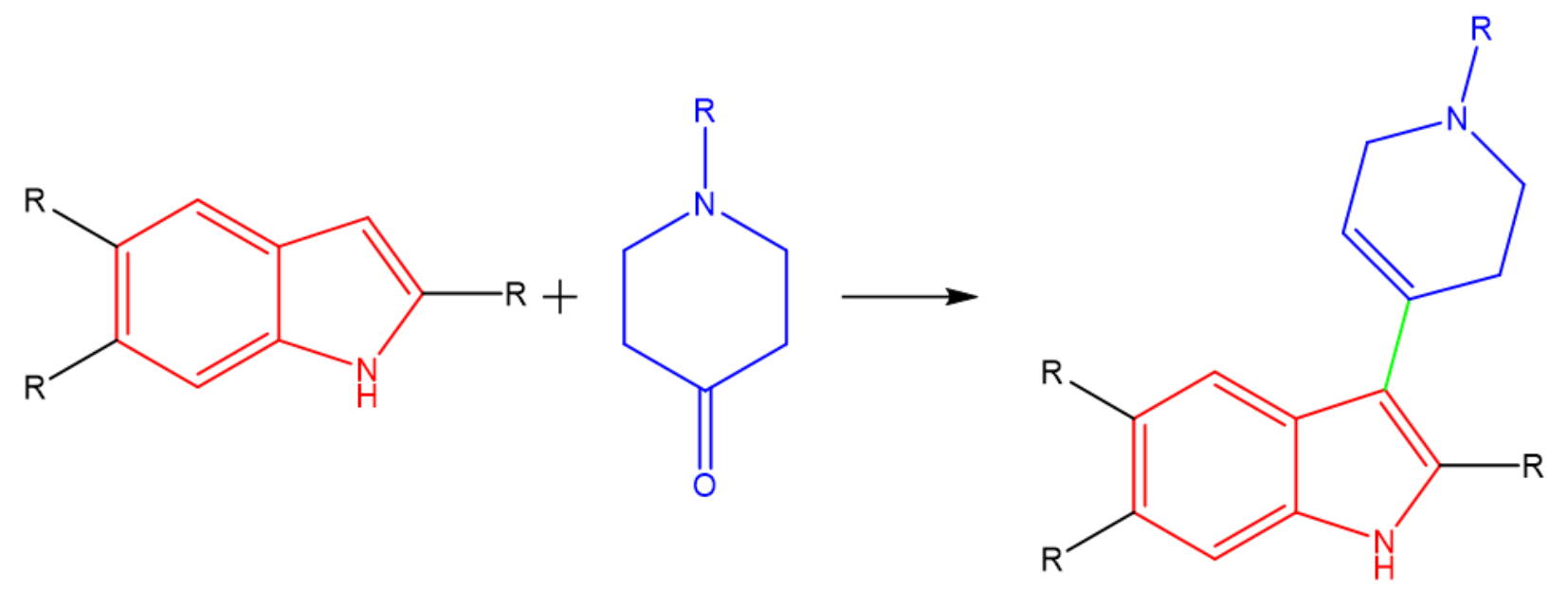

Return to Index of Reactions 


\section{Heteroatom Alkylation via Nucleophilic Attack ${ }^{1}$}

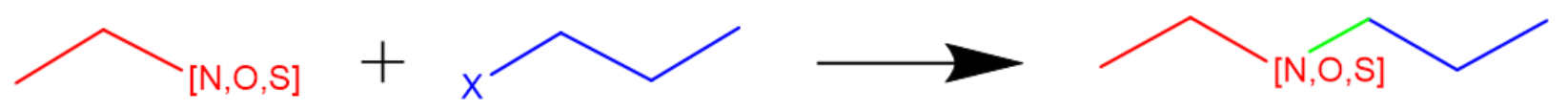

\section{Reaction Notes:}

- $\mathrm{X}$ is a halogen

- The nucleophile can be $1^{0}, 2^{0}$, or $3^{0}$.

Return to Index of Reactions 


\section{Aldol Condensation ${ }^{31,32,33}$}
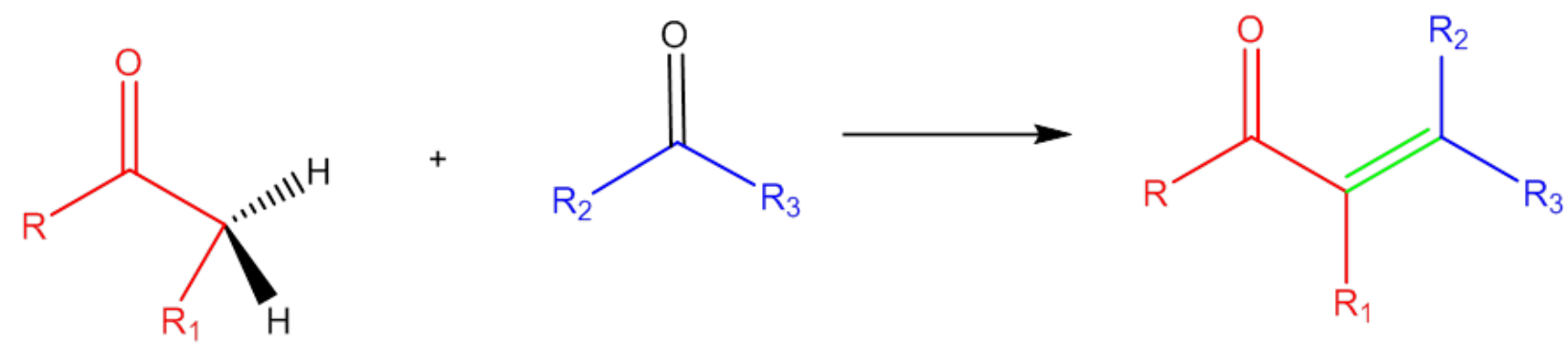

\section{Reaction Notes:}

- $\quad$ R groups can be aryl or alkyl.

Return to Index of Reactions 


\section{Sonogashira ${ }^{1,34}$}

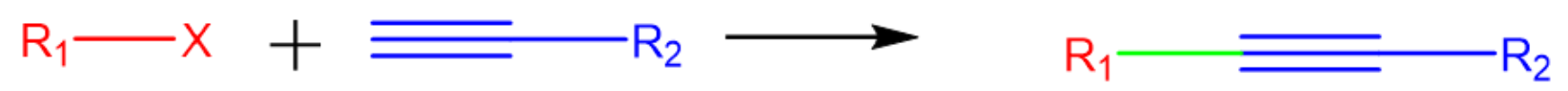

\section{Reaction Notes:}

- R1: aryl, vinyl

- X: Br, I

- R2: any $\mathrm{C}$

Return to Index of Reactions 


\section{Mizoroki-Heck Terminal Vinyl ${ }^{1}$}
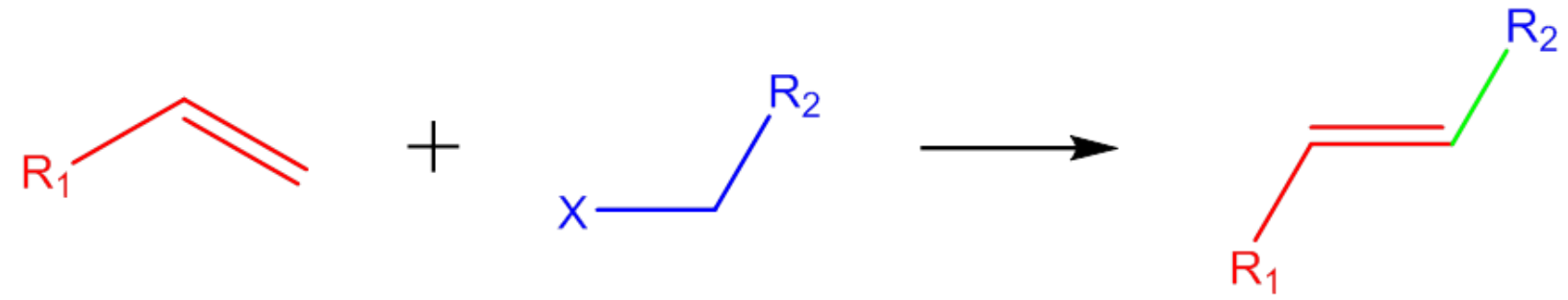

\section{Reaction Notes:}

- $\quad \mathrm{R} 1$ : aryl, $\mathrm{COO}, \mathrm{CN}$ (electron withdrawing groups yield trans selectivity)

- R2: aryl, vinyl

- $\mathrm{X}: \mathrm{Cl}, \mathrm{Br}, \mathrm{I}$

Return to Index of Reactions 


\section{Mizoroki-Heck Non-Terminal Vinyl ${ }^{1}$}
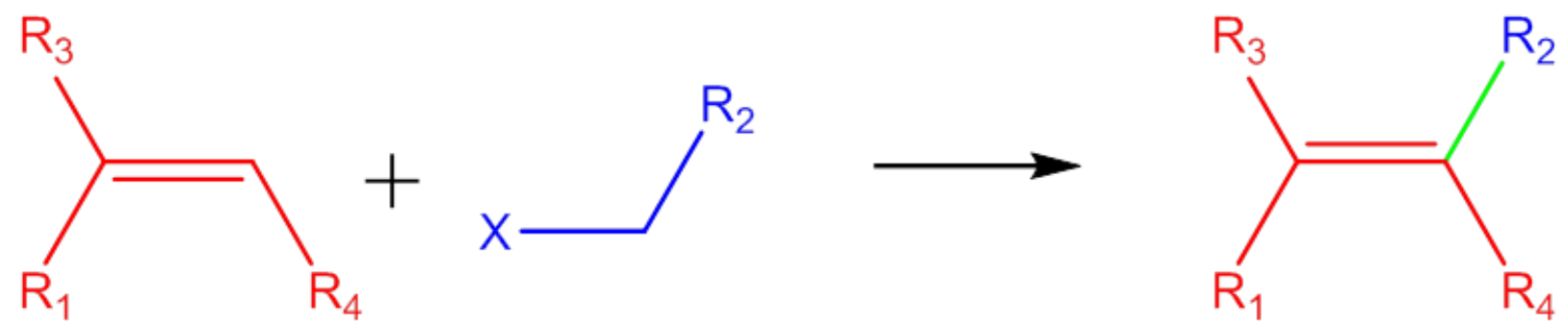

\section{Reaction Notes:}

- R1: aryl, COO, CN

- R2: aryl, vinyl

- X: Cl, Br, I

Return to Index of Reactions 


\section{Grignard Carbonyl ${ }^{1,35,36}$}

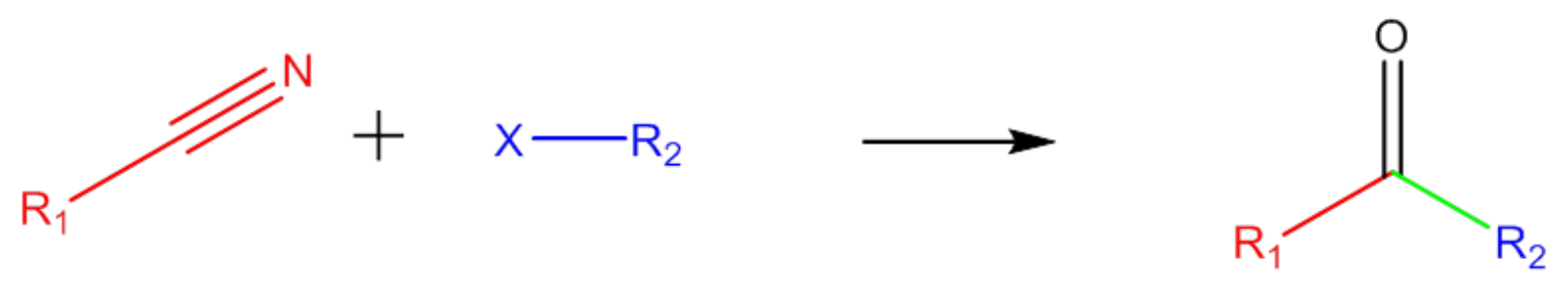

\section{Reaction Notes:}

- Reactant 2 must be transformed into Grignard $(\mathrm{RMgX})$ reagent first.

- R2: aryl, alkyl

- $\mathrm{X}: \mathrm{Cl}, \mathrm{Br}, \mathrm{I}$

Return to Index of Reactions 


\section{Grignard Alcohol ${ }^{1,37,38}$}<smiles>[R]C([R])=O</smiles>

\section{Reaction Notes:}

- Reactant 2 must be transformed into Grignard $(\mathrm{RMgX})$ reagent first.

- It is possible that this reaction will create a stereocenter.

- R2: H, C

- $\mathrm{X}: \mathrm{Cl}, \mathrm{Br}, \mathrm{I}$

Return to Index of Reactions

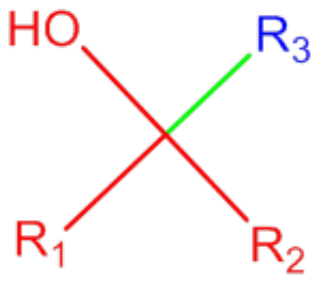




\section{Negishi $^{1,39}$}

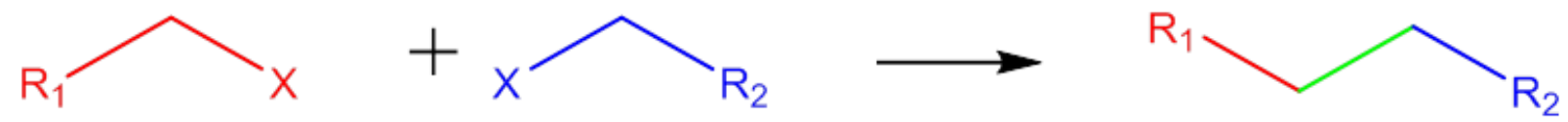

\section{Reaction Notes:}

- Formation of $\mathrm{Zn}$ halide is required as an additional step.

Return to Index of Reactions 
Wittig $^{1,40,41}$
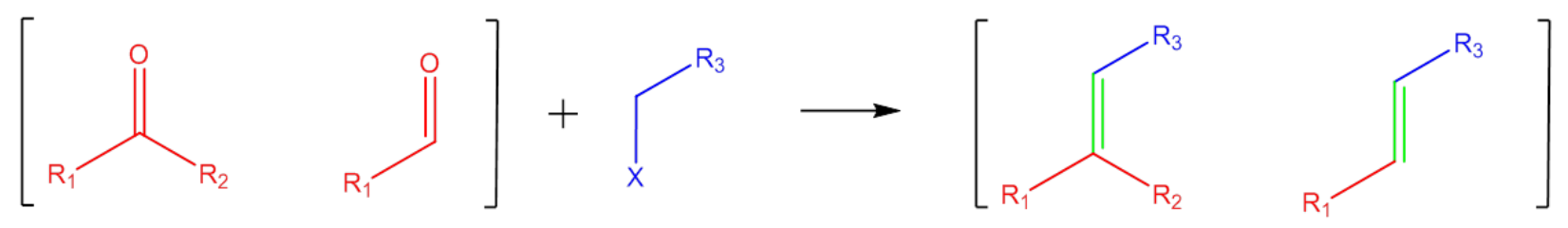

\section{Reaction Notes:}

- This reactions requires the formation of ylide from the alkyl halide by adding triaryl phosphine.

- Not stereoselective: both E/Z isomers can be formed depending on reaction conditions.

- R1 and R2: aryl, alkyl, vinyl, many functional groups tolerated here.

- $\mathrm{X}: \mathrm{Cl}, \mathrm{Br}, \mathrm{I}$

- Only primary alkyl halides allowed for second reactant.

- R3: must be a carbon, but not attached to Br, I, or OMet (eliminates ylide).

Return to Index of Reactions 


\section{Friedel-Crafts Alkylation ${ }^{42,43,44}$}
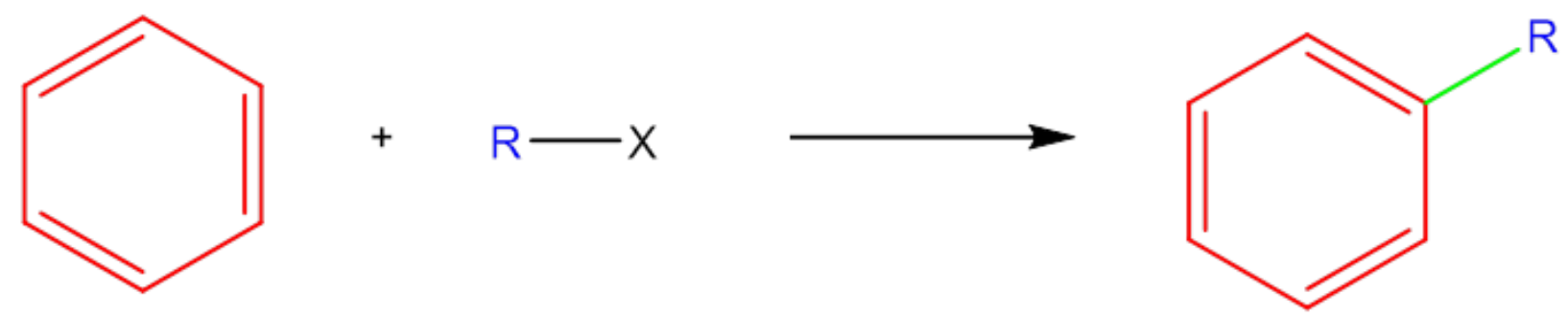

\section{Reaction Notes:}

- This reaction must utilize an arene.

- R: any alkyl group.

- Requires $\mathrm{FeCl}_{3}$ catalyst.

- $\mathrm{X}: \mathrm{Cl}, \mathrm{Br}$

Return to Index of Reactions 


\section{Friedel-Crafts Acylation ${ }^{45,46,47}$}
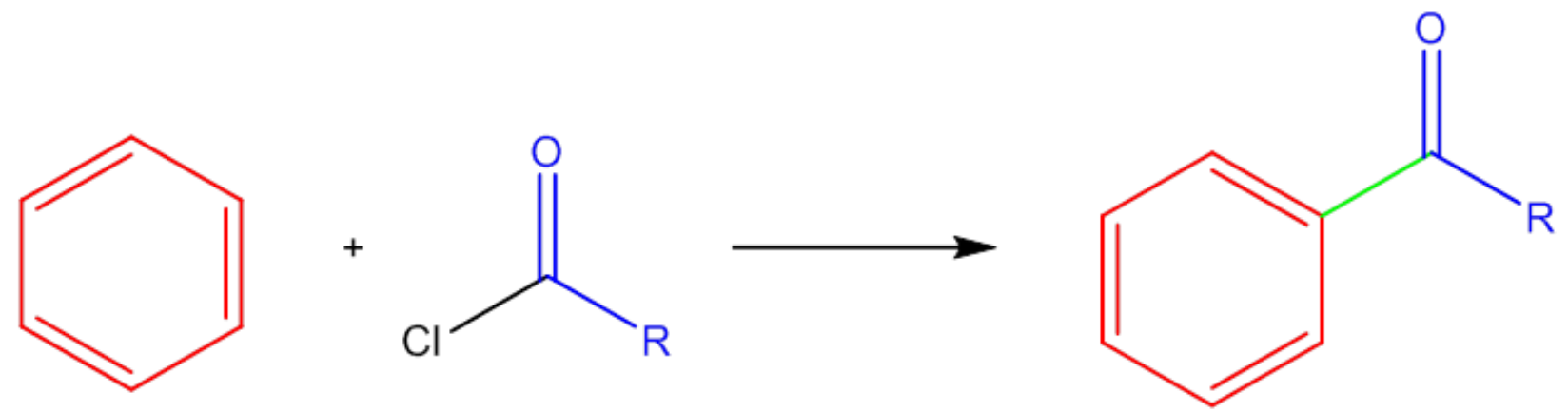

\section{Reaction Notes:}

- This reaction must utilize an arene.

- R: any alkyl group.

- Requires Lewis Acid Catalyst $\mathrm{AlCl}_{3}$.

Return to Index of Reactions 


\section{Michael-Addition ${ }^{48}$}

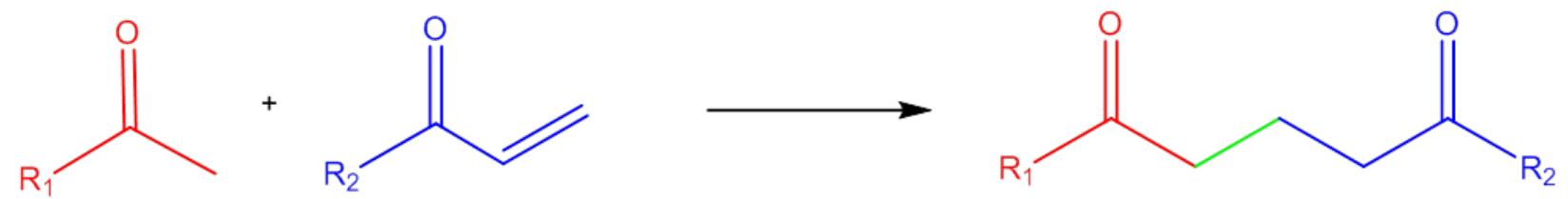

\section{Reaction Notes:}

- This reaction requires a base $(\mathrm{NaOH}$ or $\mathrm{KOH})$ to drive the reaction.

- R groups may be alkyl or aryl.

Return to Index of Reactions 


\section{Schotten-Baumann Amide ${ }^{1}$}<smiles>[R]N</smiles><smiles>[R2]NC([R1])=O</smiles><smiles>[R]C(=O)N([R2])CC</smiles>

\section{Reaction Notes:}

- Activation of carboxy group $(\mathrm{COOH} \rightarrow \mathrm{COCl})$ required as additional step.

- R1, R2, and R3: aryl allowed

Return to Index of Reactions 


\section{Sulfonamide $^{1}$}

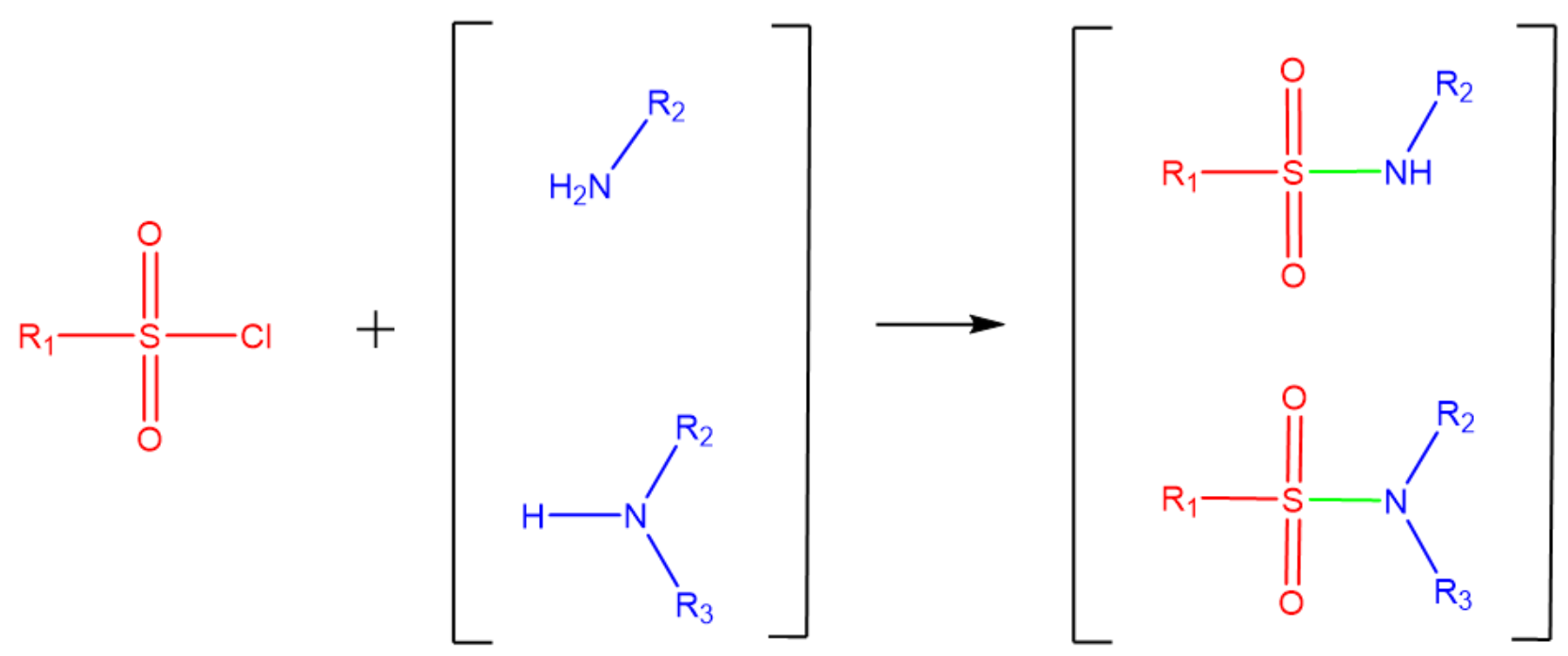

\section{Reaction Notes:}

- R1: C, N

Return to Index of Reactions 


\section{Wöhler $^{1}$}

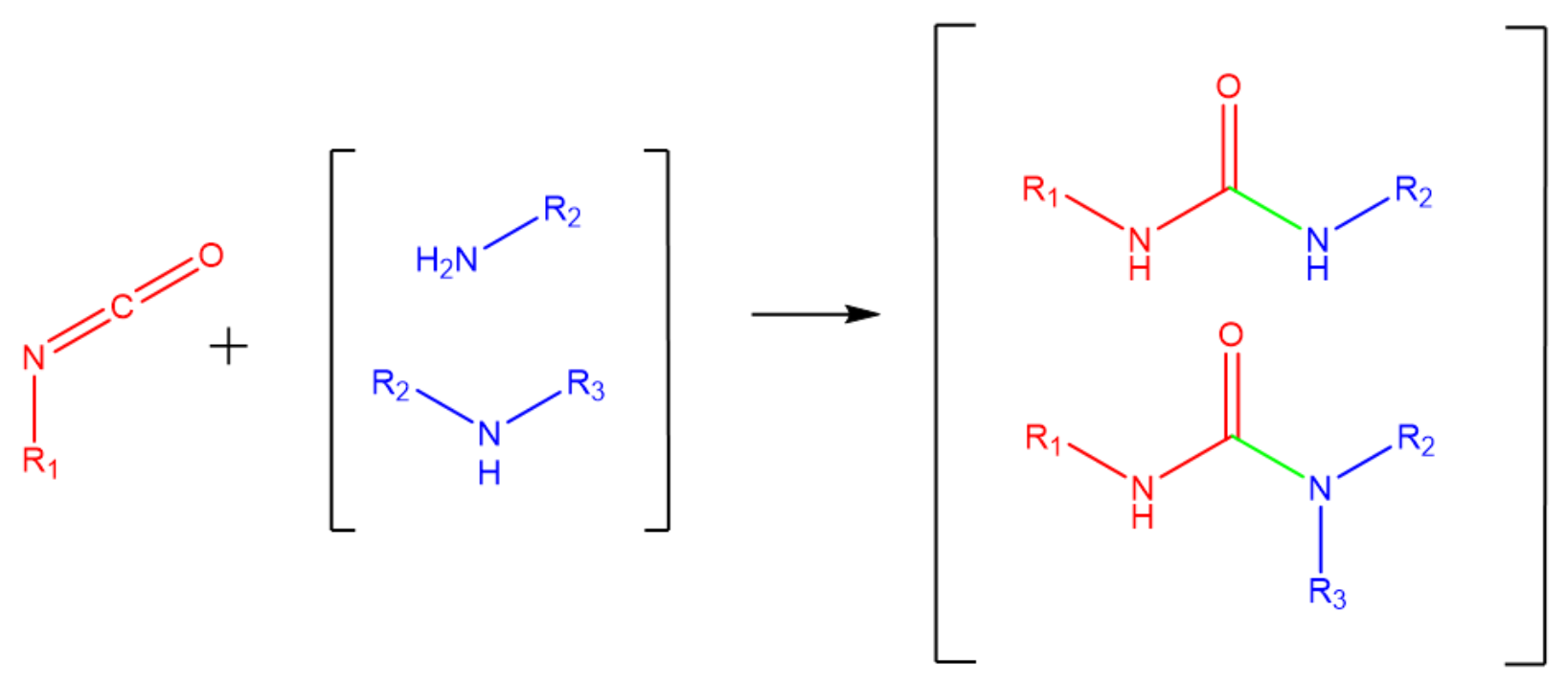

\section{Reaction Notes:}

- R1: C, aryl, alkyl

- R2: C, aryl, alkyl

- R3: C, aryl, alkyl

Return to Index of Reactions 


\section{Thiourea via Nucleophilic Addition ${ }^{1}$}

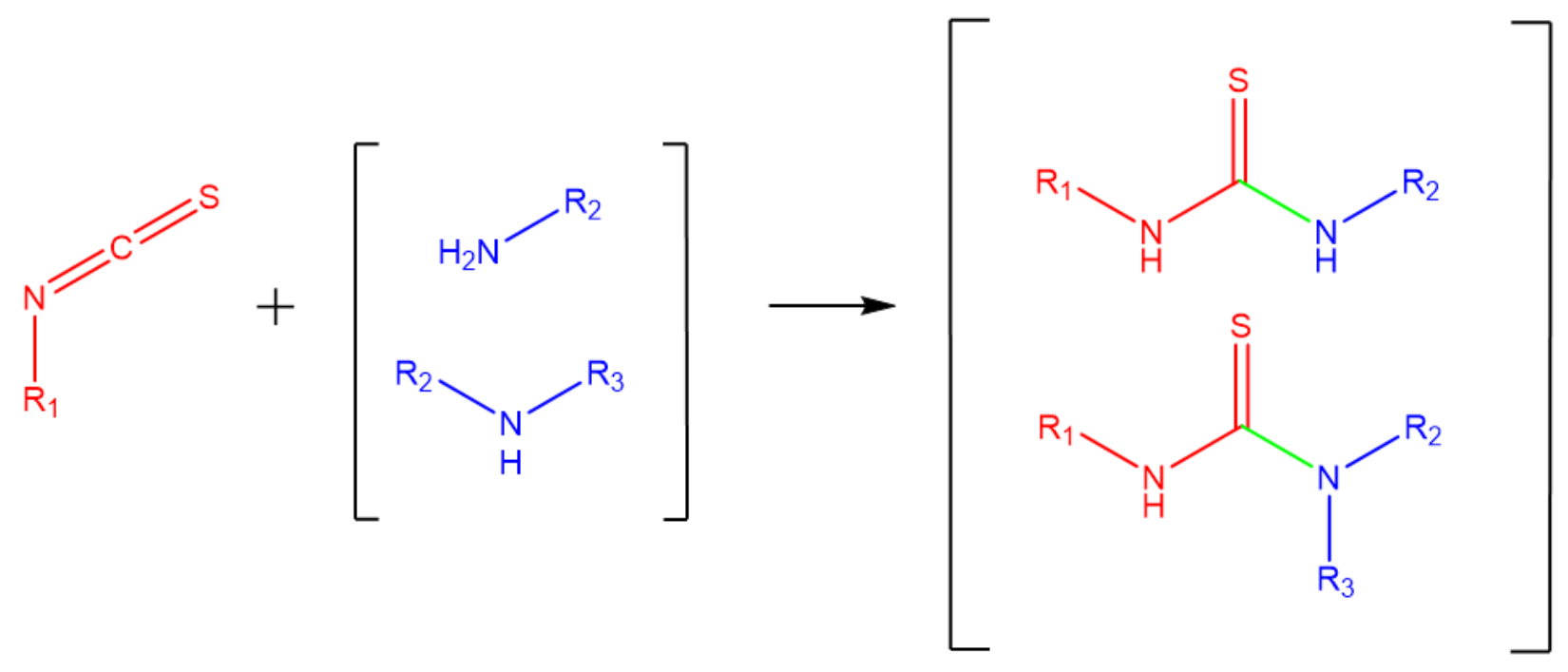

\section{Reaction Notes:}

- R1: C, aryl, alkyl

- R2: C, aryl, alkyl

- R3: C, aryl, alkyl

Return to $\underline{\text { Index of Reactions }}$ 


\section{NHS Ester Reaction ${ }^{49}$}<smiles>[R]C(=O)NCCC</smiles>

\section{Reaction Notes:}

- Requires the NHS (N-Hydroxysuccinimide) ester reagent.

- The R-group needs to start with an oxygen or carbon.

- The amine needs to be a primary amine.

Return to Index of Reactions 


\section{Heteroaromatic via Nucleophilic Substitution ${ }^{1,50}$}

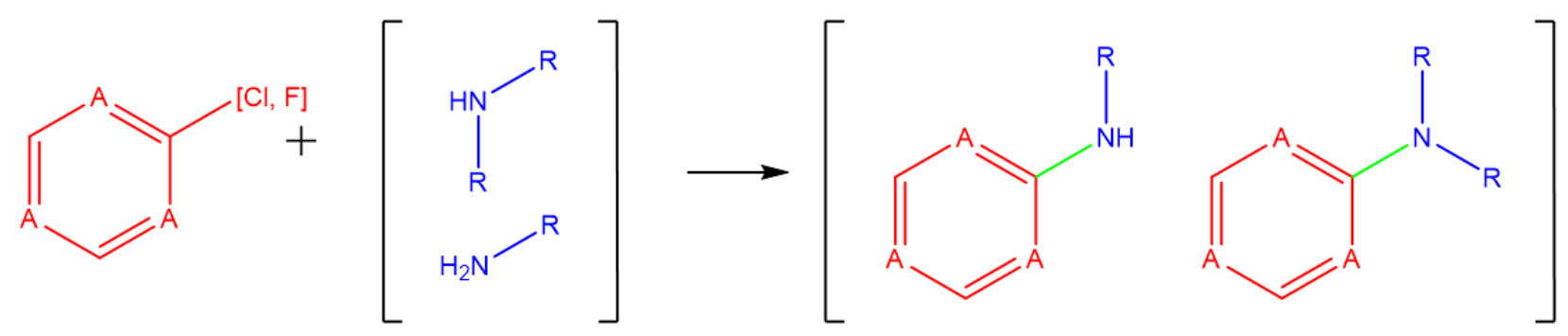

\section{Reaction Notes:}

- The heteroatoms activate the para and ortho positions for substitution.

- A: C or $\mathrm{N}$ of a pyridine, pyrimidine, or triazine.

- The $\mathrm{N}$ cannot be double bonded, triple bonded, or bonded to 3 heavy atoms.

Return to Index of Reactions 


\section{Ortho Substituted Nitroaniline via Nucleophilic Substitution ${ }^{1,50}$}

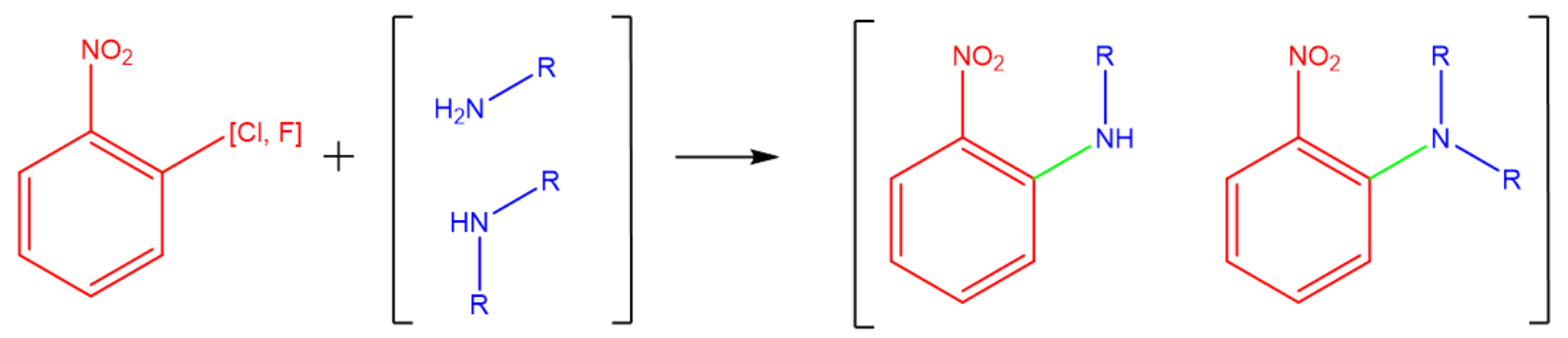

\section{Reaction Notes:}

- The ortho nitro group has an activating effect on nucleophilic substitution.

- The $\mathrm{N}$ must not be charged, double bonded, or triple bonded.

Return to Index of Reactions 


\section{Para Substituted Nitroaniline via Nucleophilic Substitution ${ }^{1}$}

$\longrightarrow$

\section{Reaction Notes:}

- The para nitro group has an activating effect on nucleophilic substitution.

- The N must not be charged, double bonded, or triple bonded.

Return to Index of Reactions 


\section{Weinreb-Nahm Amide ${ }^{51,52}$}
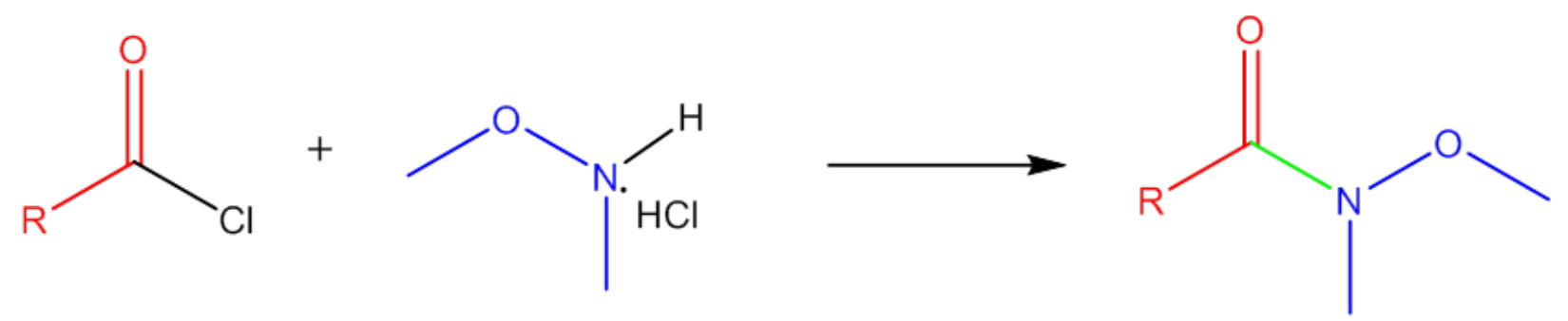

\section{Reaction Notes:}

- $\quad$ R: aryl or alkyl.

Return to Index of Reactions 


\section{Staudinger Reaction ${ }^{53,54}$}

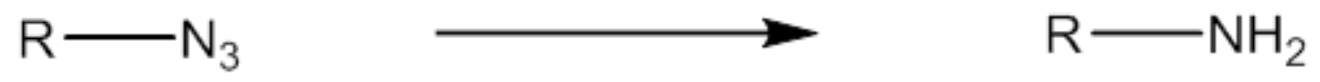

\section{Reaction Notes:}

- Addition of $\mathrm{PPh}_{3}$ is required to react with the azide.

- $\quad$ R: any alkyl or aryl group.

Return to Index of Reactions 


\section{Beckmann Rearrangement $^{55,56}$}

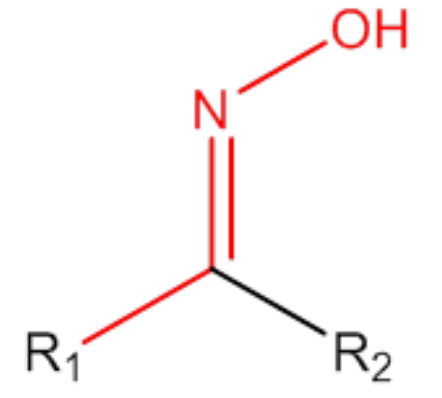

\section{Reaction Notes:}

- Amide synthesis via oxime rearrangement.

- Requires acid catalyst $\mathrm{H}_{2} \mathrm{SO}_{4}$.

Return to Index of Reactions

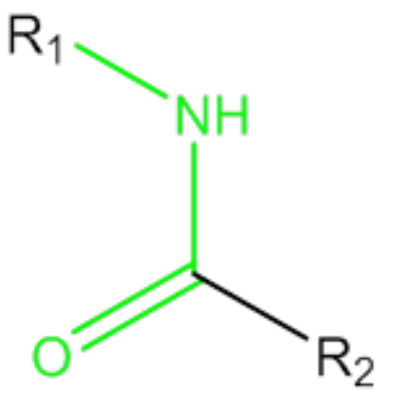




\section{Amide Reduction $^{57,58}$}
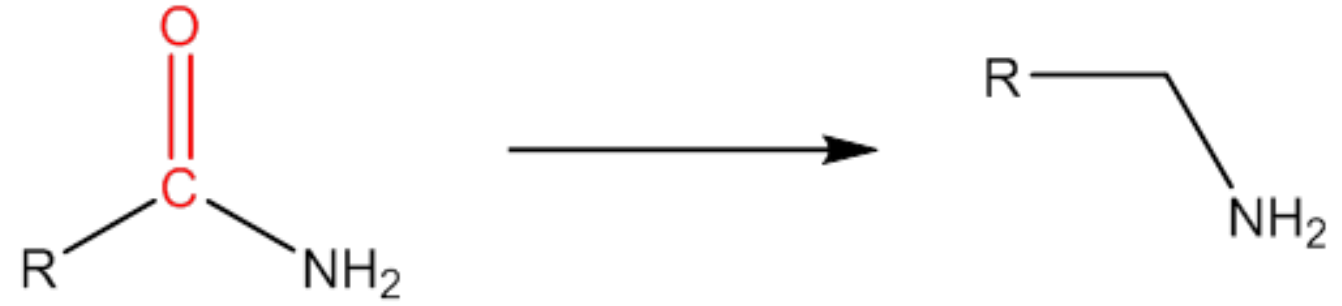

Return to Index of Reactions 


\section{Imine Reduction $^{59,60}$}
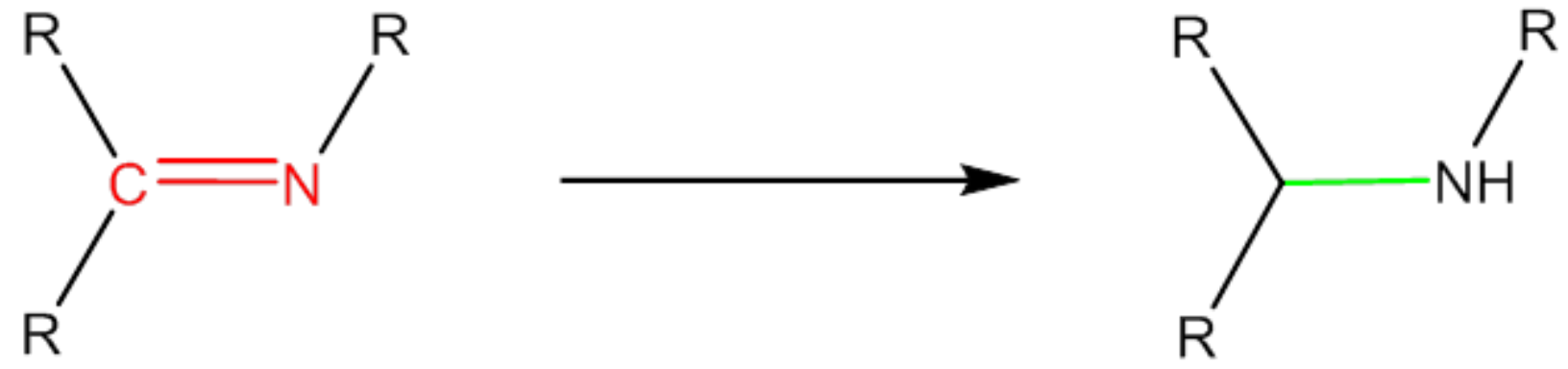

Return to Index of Reactions 
Nitrile Reduction $^{61}$
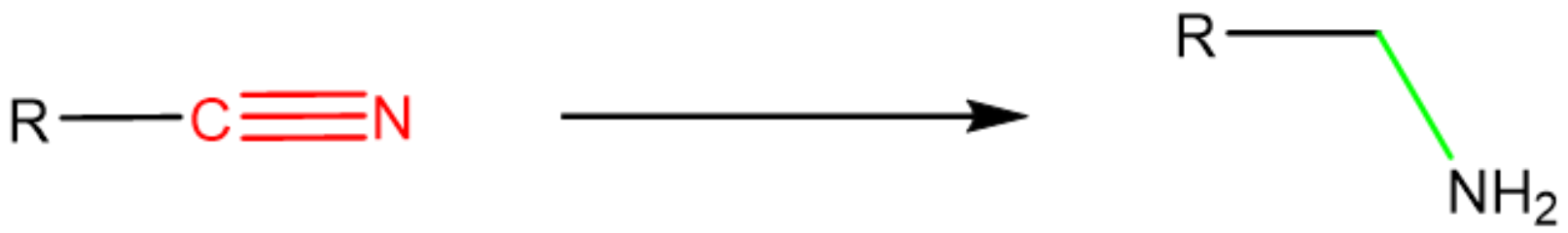

Return to Index of Reactions 


\section{Ester Reduction $^{62}$}
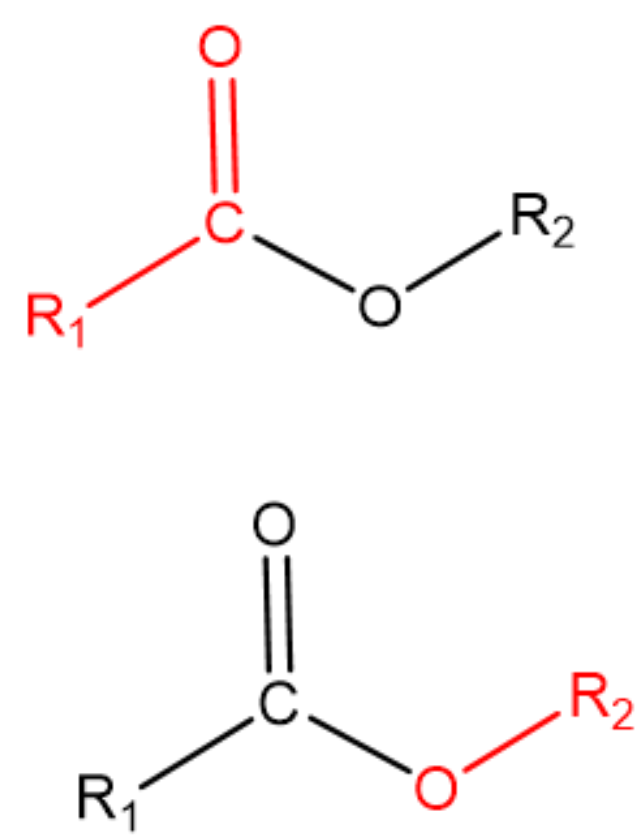

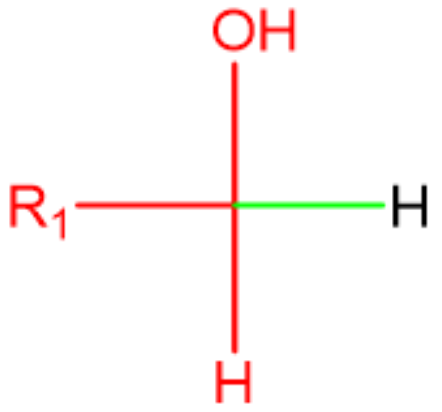

$\mathrm{HO}-\mathrm{R}_{2}$

Both products are formed; however, only one is randomly selected to be included in the synthesis.

Return to Index of Reactions 


\section{Ketone Reduction ${ }^{63,64}$}
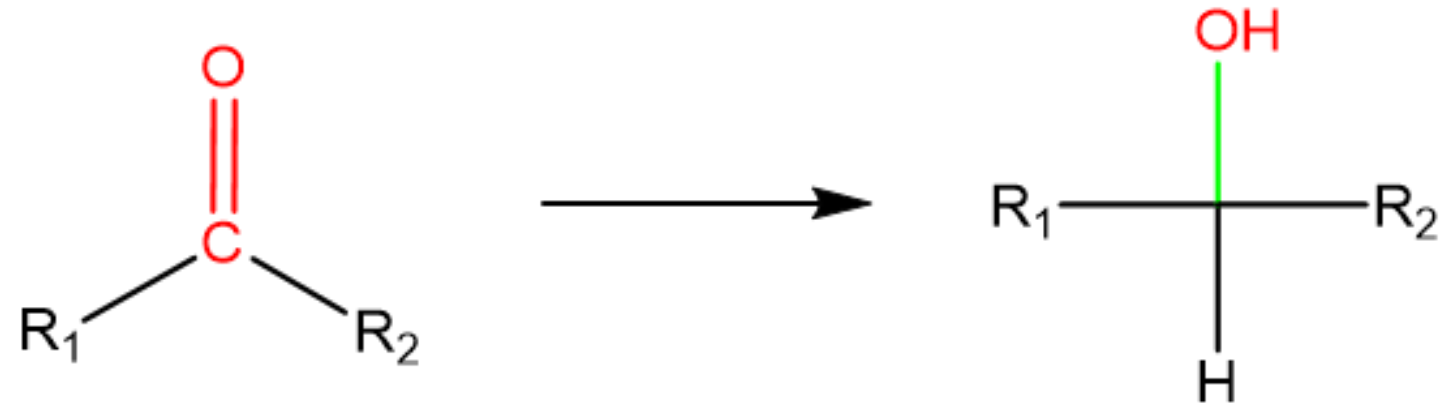

Return to Index of Reactions 


\section{Alkene Reduction ${ }^{65}$}
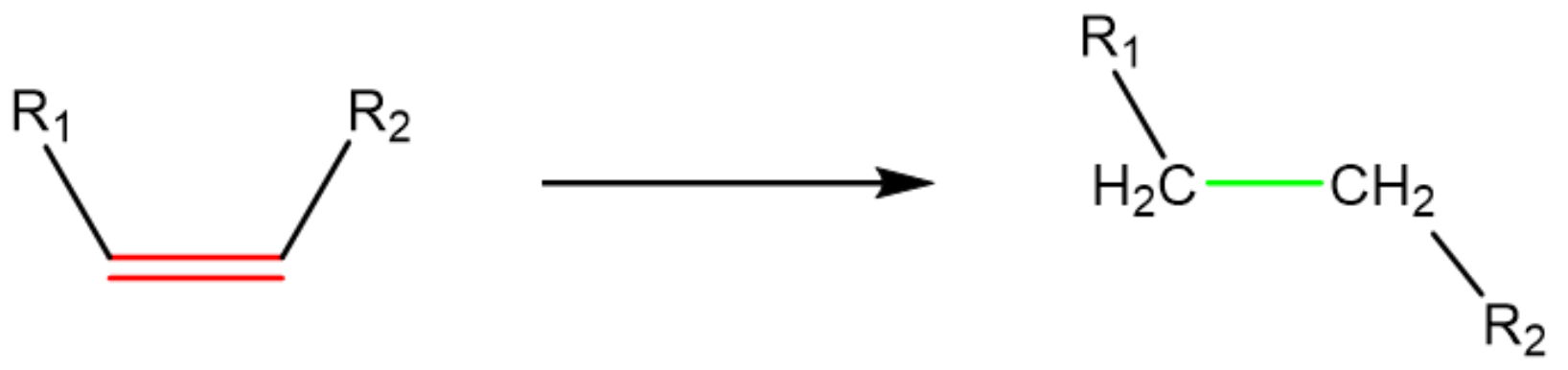

Return to Index of Reactions 


\section{Alkyne Reduction}
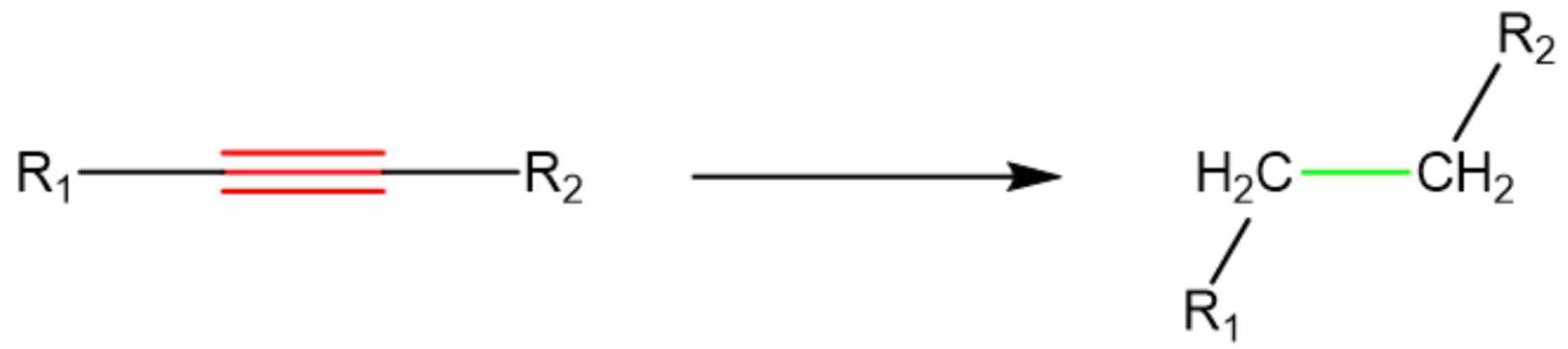

Return to Index of Reactions 
Nitroso_Reduction ${ }^{66,67}$
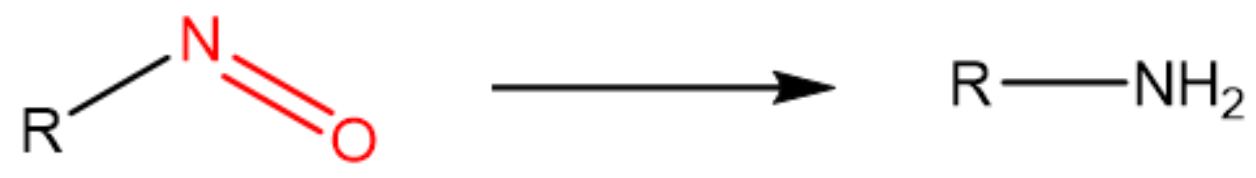

Return to Index of Reactions 
Nitro to Hydroxylamine ${ }^{68,69}$
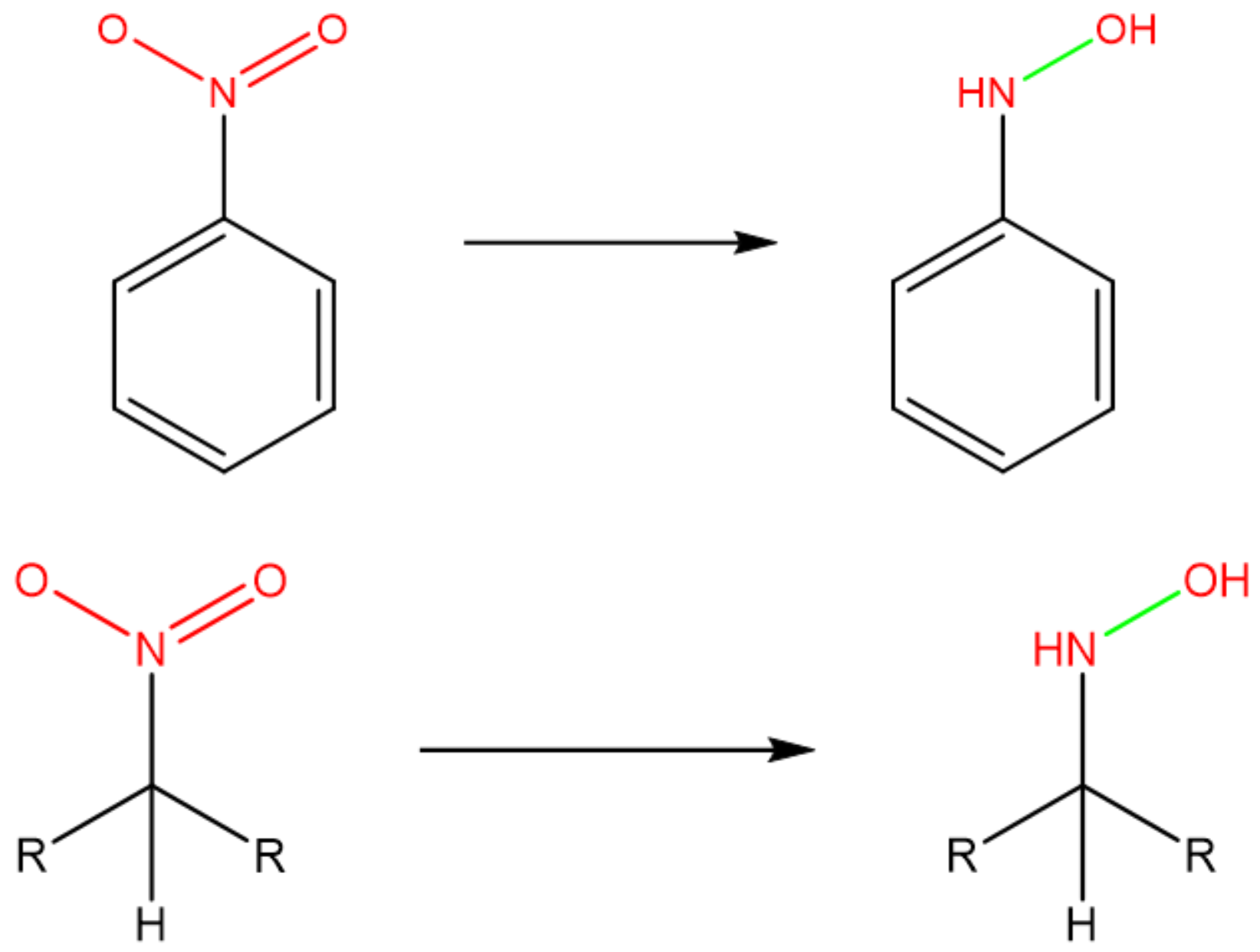

Return to Index of Reactions 
Nitro to Amine/Aniline $e^{70,71}$
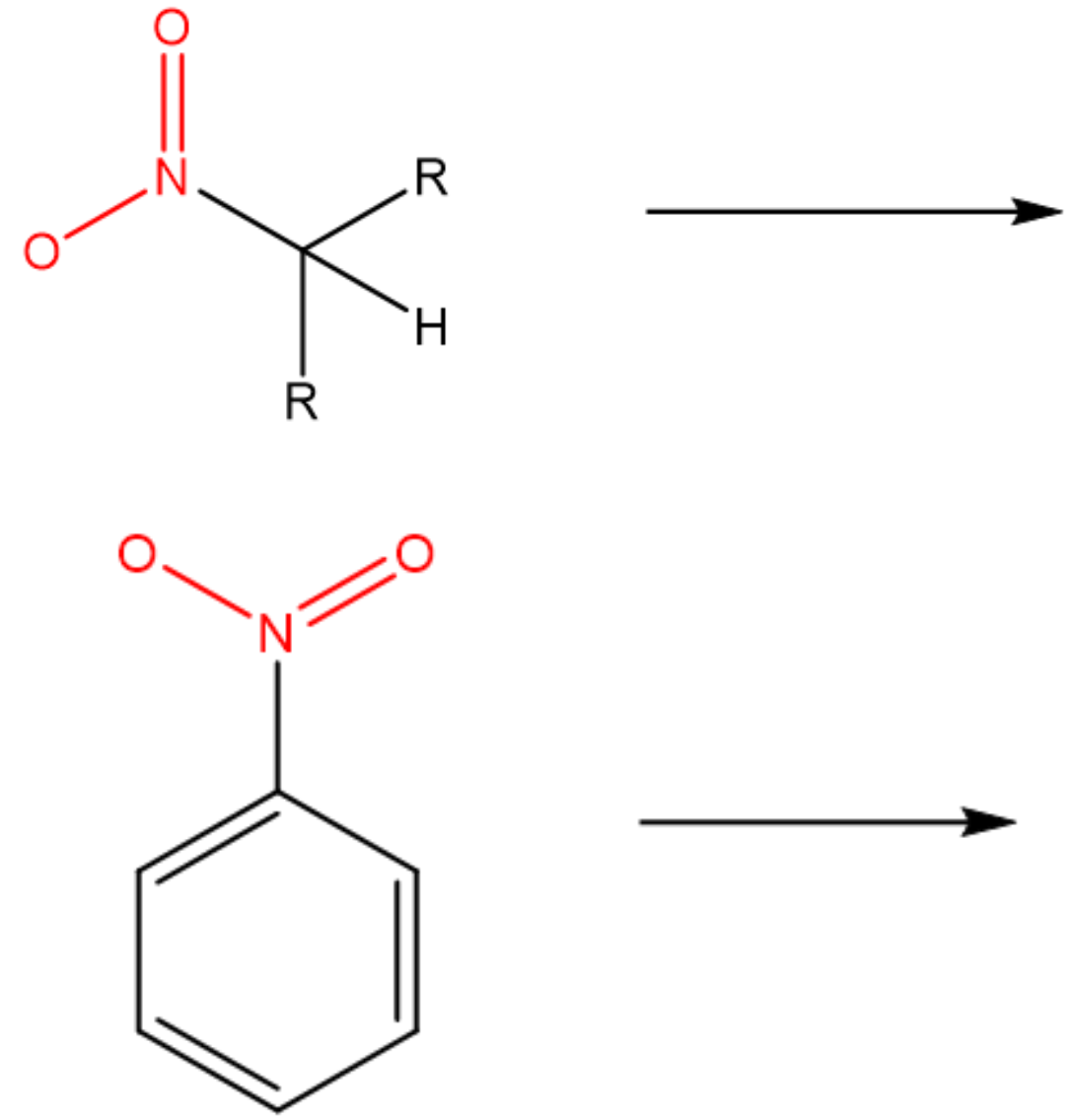
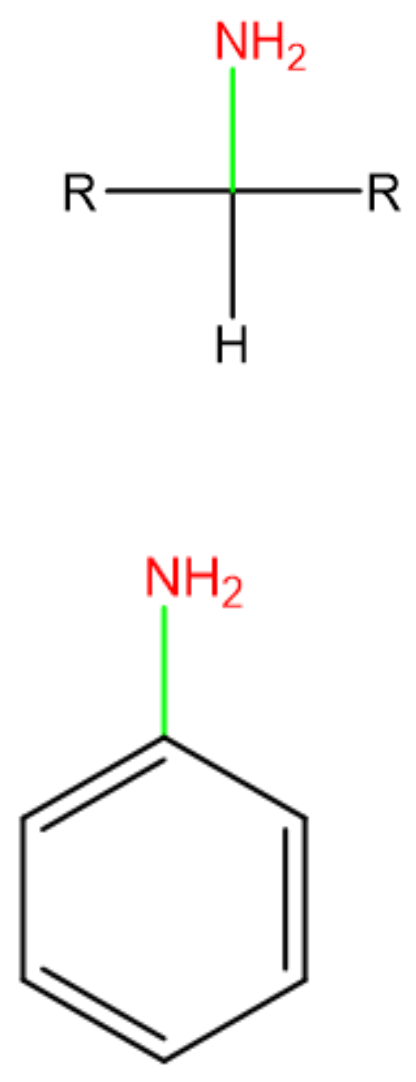

Return to Index of Reactions 


\section{Aliphatic Nitro to Oxime ${ }^{72,73}$}
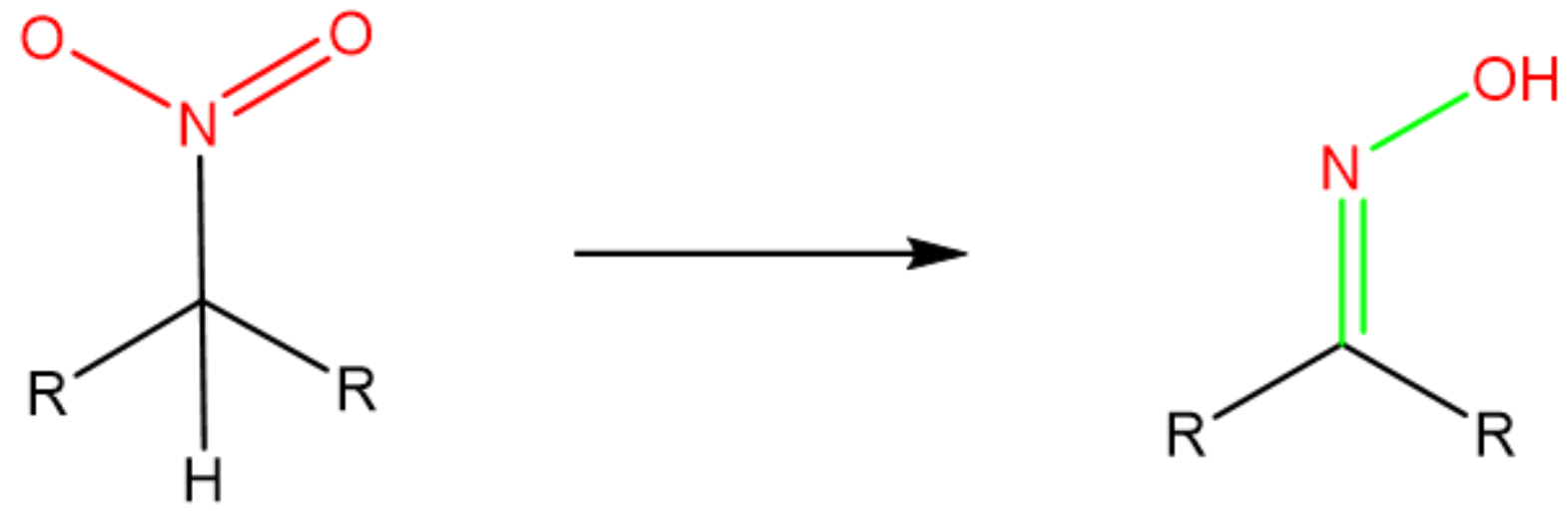

Return to Index of Reactions 


\section{Aliphatic Nitro to Hydrocarbon ${ }^{74,75}$}
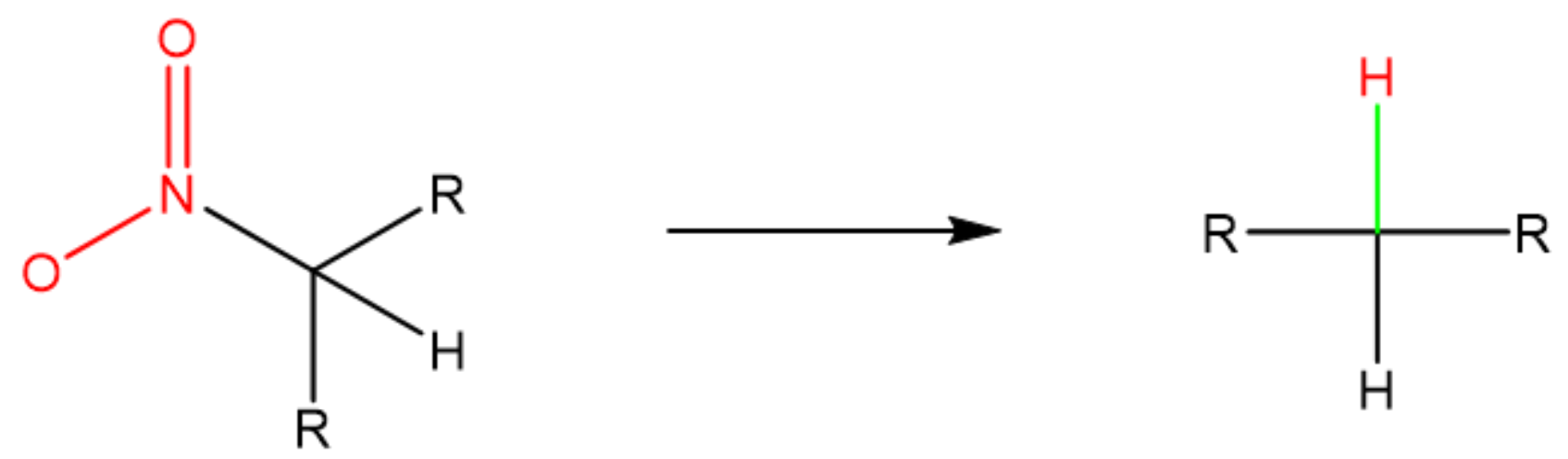

Return to Index of Reactions 


\section{Primary Alcohol [O] to Aldehyde ${ }^{57,76}$}
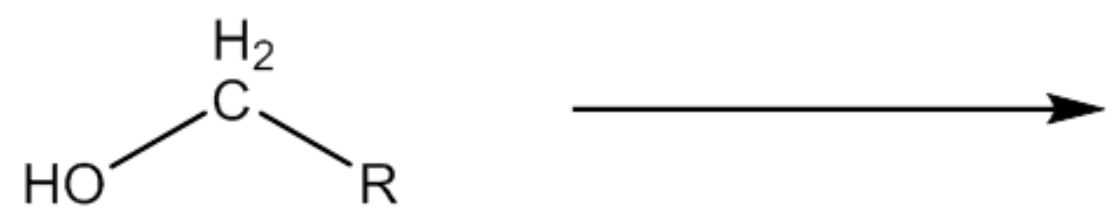

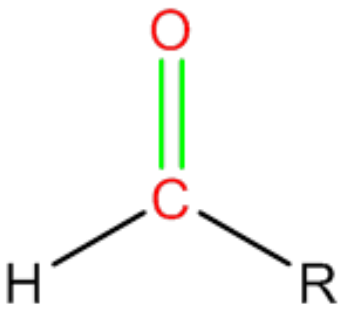

Return to Index of Reactions 


\section{Primary Alcohol [O] to Carboxylic Acid ${ }^{57,77}$}
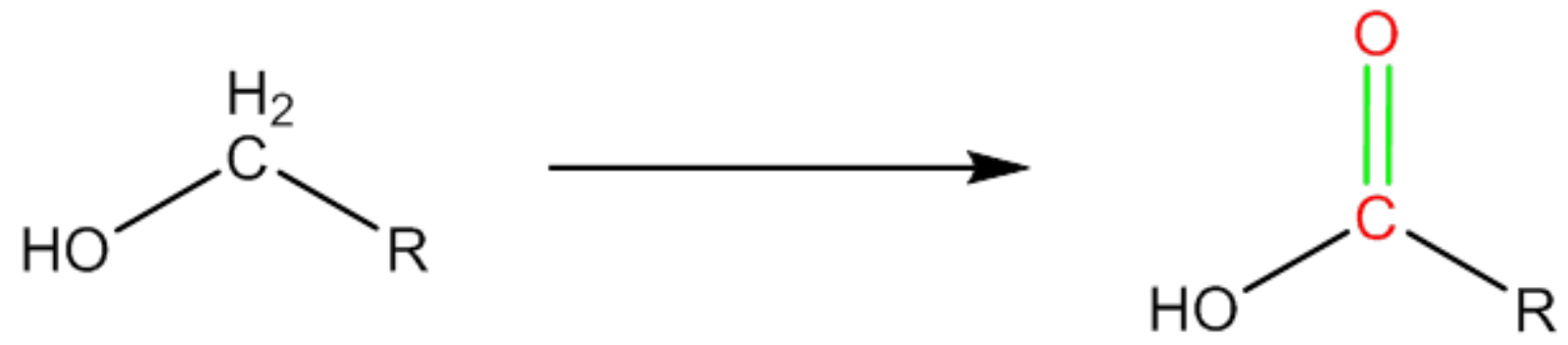

Return to Index of Reactions 


\section{Secondary Alcohol [O] to Ketone ${ }^{57,78}$}
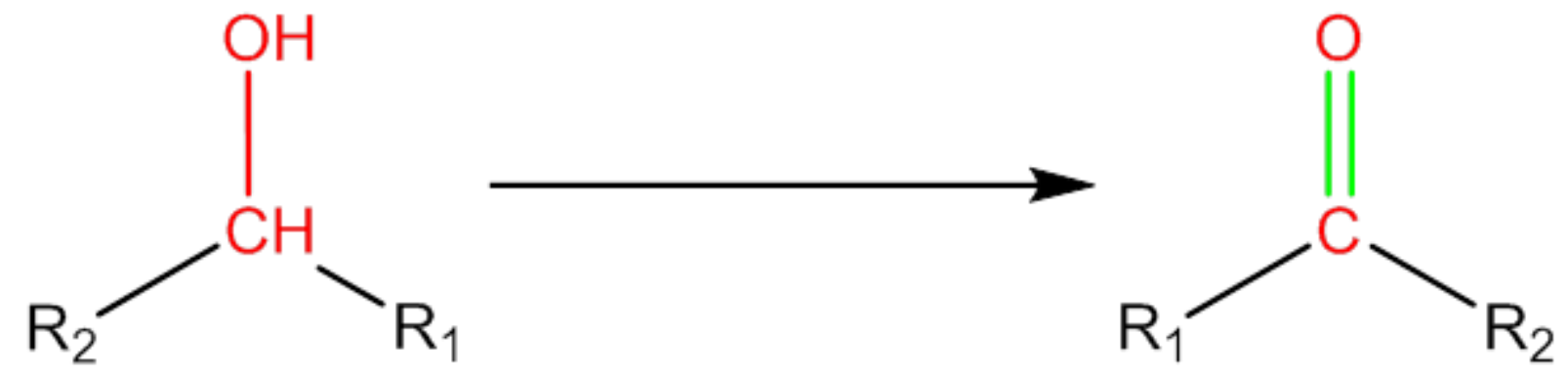

Return to Index of Reactions 


\section{Sulfide to Sulfoxide ${ }^{79,80}$}
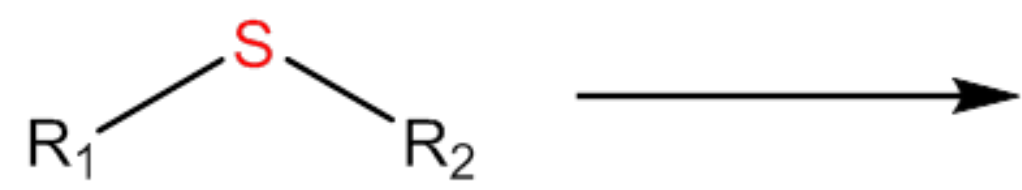

Return to Index of Reactions

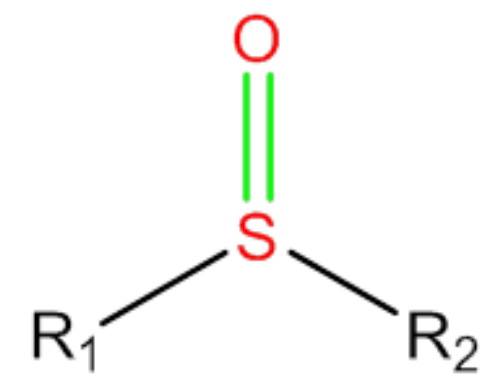




\section{Sulfide to Sulfone ${ }^{81}$}
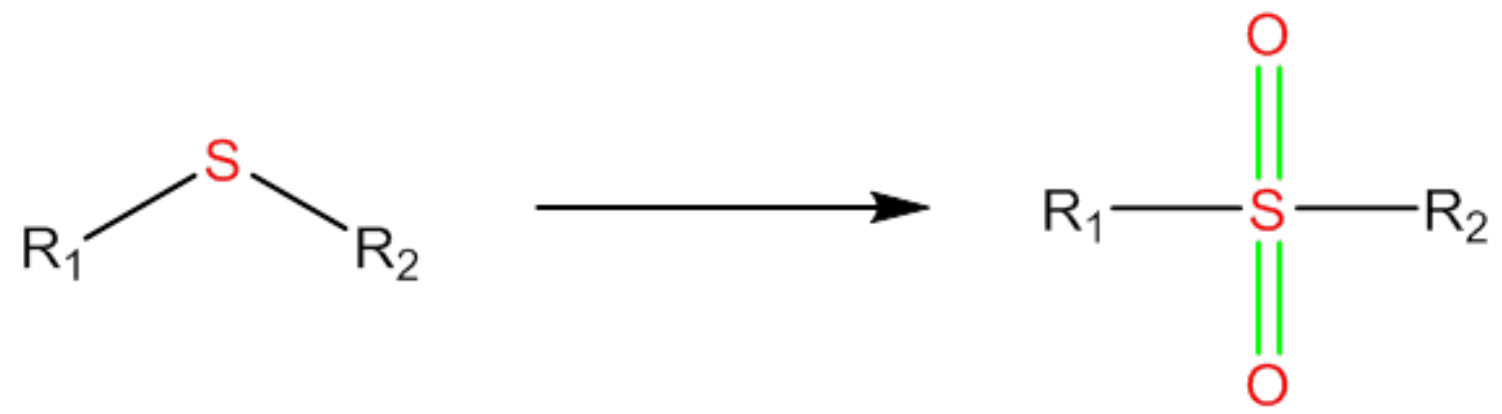

Return to Index of Reactions 


\section{Sulfoxide to Sulfone ${ }^{81}$}
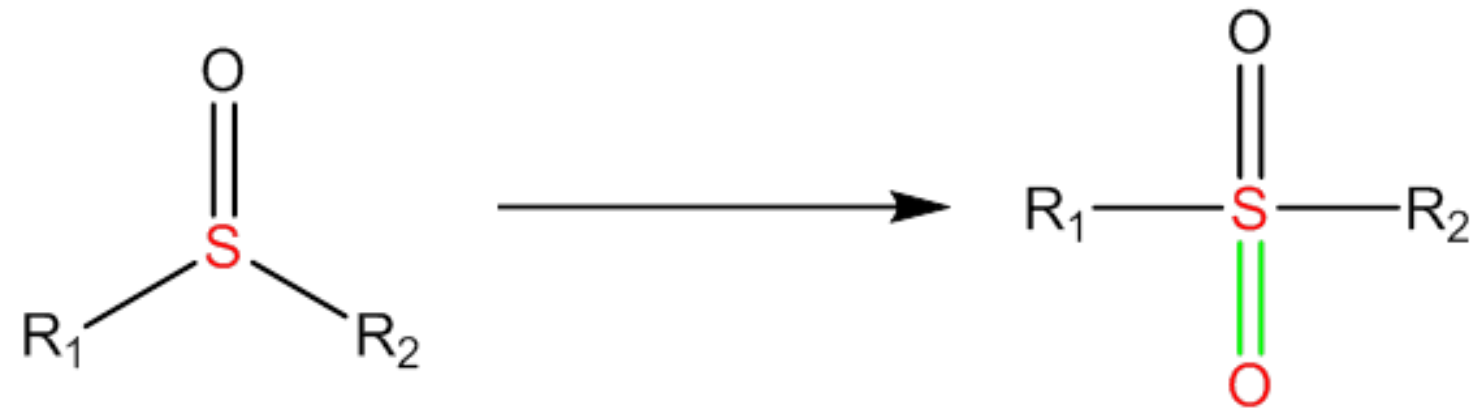

Return to Index of Reactions 


\section{REFERENCES}

1 Hartenfeller, M.; Eberle, M.; Meier, P.; Nieto-Oberhuber, C.; Altmann, K.H.; Schneider, G.; Jacoby, E.; Renner, S. A Collection of Robust Organic Synthesis Reactions for In Silico Molecule Design J. Chem. Inf. Model. 2011, 51, 3093-3098

2 Wikipedia. http://en.wikipedia.org/wiki/Pictet-Spengler_reaction (accessed Mar 28, 2016)

3 The Organic Synthesis Archive. http://www.synarchive.com/named-reactions/PictetSpengler_Reaction (accessed Mar 28, 2016)

4 Named Reaction. http://www.name-reaction.com/pictet-spengler-reaction (accessed Mar 28, 2016)

5 Sharma, A.; Singh, M.; Rai, N.N.; Sawant, D. Mild and Efficient Cyanuric Chloride Catalyzed Pictet-Spengler Reaction. Beilstein J. Org. Chem. 2013, 9, 1235-1242. For Online version, see: http://www.ncbi.nlm.nih.gov/pmc/articles/PMC3701375/ (accessed Mar 28, 2016)

6 Maresh, J.J.; Giddings, L.A.; Friedrich, A.; Loris, E.A.; Panjikar, S.; Trout, B.L. Stockigt, J.; Peters, B.; O’Connor, S.E. Strictosidine Synthase: Mechanism of a Pictet-Spengler Catalyzing Enzyme. J. Am. Chem. Soc., 2008, 130, 710-723. For Online version, see: http://www.ncbi.nlm.nih.gov/pmc/articles/PMC3079323/ (accessed Mar 28, 2016)

7 Mittal, N.; Sun, D.X.; Seidel, D. Conjugate-base-stabilized Brønsted acids: Catalytic Enantioselective Pictet-Spengler Reactions with Unmodified Tryptamine. Org Lett., 2014, $16,1012-5$.

8 Organic Chemistry Portal. http://www.organicchemistry.org/synthesis/heterocycles/imidazoles.shtm (accessed Mar 28, 2016)

9 Molander, G.A.; Ajayi, K. Oxidative Condensations to Form Benzimidazole-Substituted Patassium Organotrifluoroborates. Org. Lett., 2012, 14, 4242-45. For Online version, see: http://www.ncbi.nlm.nih.gov/pmc/articles/PMC3428750/ (accessed Mar 28, 2016)

10 Nannapaneni, D.T.; Gupta, A.; Reddy, M.I.; Sarva, R.C. Synthesis, Characterization, and Biological Evaluation of Benzimidazole Derivatives as Potential Anxiolytics. J. Young Pharm., 2010, 2, 273-9. For Online version, see: http://www.ncbi.nlm.nih.gov/pmc/articles/PMC2964780/ (accessed Mar 28, 2016)

11 Wikipedia. http://en.wikipedia.org/wiki/Niementowski_quinoline_synthesis (accessed Mar 28, 2016)

12 Berg, R.; Straub, B.F.; Advancements in the Mechanistic Understanding of the CopperCatalyzed Azide-Alkyne Cycloaddition. Beilstein J. Org. Chem., 2013, 9, 2715-50. For Online version, see: http://www.ncbi.nlm.nih.gov/pmc/articles/PMC3869285/ (accessed Mar 28, 2016)

13 Ortega-Arizmendi, A.I.; Aldeco-Perez, E.; Cuevas-Yanez, E. Alkyne-Azide Cycloaddition Catalyzed by Silver Chloride and "Abnormal" Silver N-Heterocyclic Carbene Complex. Sci. World J., 2013, 2013, 186537. For Online version, see: http://www.ncbi.nlm.nih.gov/pmc/articles/PMC3836375/ (accessed Mar 28, 2016)

14 Thompson, A. S.; Humphrey, G. R.; DeMarco, A. M.; Mathre, D. J.; Grabowski, E. J. Direct Conversion of Activated Alcohols to Azides Using Diphenyl Phosphorazidate. A Practical Alternative to Mitsunobu Conditions. J. Org. Chem., 1993, 58, 5886-5888.

15 Kupracz, L.; Hartwig, J.; Wegner, J.; Ceylan, S.; Kirschning, A. Multistep Flow Synthesis of Vinyl Azides and their use in the Copper-Catalyzed Huisgen-type Cycloaddition under 
Inductive-heating Conditions. Beilstein J. Org. Chem., 2011, 7, 1441-8. For Online version, see: http://www.ncbi.nlm.nih.gov/pmc/articles/PMC3205773/ (accessed Mar 28, 2016)

16 Wikipedia. http://en.wikipedia.org/wiki/Paal\%E2\%80\%93Knorr_synthesis (accessed Mar 28, 2016)

17 Amarnath, V.; Amarnath, K. Intermediates in the Paal-Knorr Synthesis of Furans. J. Org. Chem., 1995, 60, 301-7.

18 Campaigne, E.; Foye, W.O. The Synthesis of 2,5-Diarylthiophenes. J. Org. Chem., 1952, 17, 1405-1412.

19 Wikipedia. http://en.wikipedia.org/wiki/Friedl\%C3\%A4nder_synthesis (accessed Mar 28, 2016)

20 Wikipedia. http://en.wikipedia.org/wiki/Larock_indole_synthesis (accessed Mar 28, 2016)

21 Named Reaction. http://www.organic-chemistry.org/namedreactions/click-chemistry.shtm (accessed Mar 28, 2016)

22 Himo, F.; Lovell, T.; Hilgraf, R.; Rostovtsev, V.V.; Noodleman, L.; Sharpless, K.B.; Fokin, V.V. Copper(I)-Catalyzed Synthesis of Azoles. DFT Study Predicts Unprecedented Reactivity and Intermediates. J. Am. Chem. Soc. 2005, 127, 210-216.

23 Named Reaction. http://www.organic-chemistry.org/namedreactions/pechmanncondensation.shtm (accessed Mar 28, 2016)

24 Wikipedia. http://en.wikipedia.org/wiki/Aripiprazole (accessed Mar 28, 2016)

25 Wikipedia. https://en.wikipedia.org/wiki/Dieckmann_condensation (accessed Mar 28, 2016)

26 Domling, A.; Wang, K.; Wang, W. Chemistry \& Biology Of Multicomponent Reactions. Chem. Rev., 2012, 112, 3083-3135. For Online version, see: http://www.ncbi.nlm.nih.gov/pmc/articles/PMC3712876/ (accessed Mar 28, 2016)

27 The Organic Synthesis Archive. http://www.synarchive.com/namedreactions/Dieckmann_Condensation (accessed Mar 28, 2016)

28 Goossen, L.J.; Rodriguez, N.; Melzer, B.; Linder, C.; Deng, G.; Levy, L.M. Biaryl Synthesis via Pd-Catalyzed Decarboxylative Coupling of Aromatic Carboxylates with Aryl Halides. J. Am. Chem. Soc. 2007, 129, 4824-4833.

29 Wikipedia. http://en.wikipedia.org/wiki/Stille_reaction (accessed Mar 28, 2016)

30 Lam, P. Y.; Clark, C. G.; Saubern, S.; Adams, J.; Winters, M. P.; Chan, D. M.; Combs, A. New Aryl/Heteroaryl CN Bond Cross-coupling via Arylboronic Acid/Cupric Acetate Arylation. Tet. Lett. 1998, 39, 2941-2944.

31 Wikipedia. http://en.wikipedia.org/wiki/Aldol_condensation (accessed Mar 28, 2016)

32 Liao, Y.; Xing, C.; Israel, M.; Hu, Q. Sequential Aldol Condensation - Transition MetalCatalyzed Addition Reactions of Aldehydes, Methyl Ketones and Arylboronic Acids. Org. Lett., 2011, 13, 2058-61. For Online version, see: http://www.ncbi.nlm.nih.gov/pmc/articles/PMC3077042/ (accessed Mar 28, 2016)

33 Wang, C.; Liu, J.; Leng, W.; Gao, Y. Rapid and Efficient Functionalized Ionic LiquidCatalyzed Aldol Condensation Reactions Associated with Microwave Irradiation. Int. J. Mol. Sci., 2014, 15, 1284-99. For Online version, see: http://www.ncbi.nlm.nih.gov/pmc/articles/PMC3907869/ (accessed Mar 28, 2016)

34 Wikipedia. http://en.wikipedia.org/wiki/Sonogashira_coupling (accessed Mar 28, 2016)

35 Named Reaction. http://www.organic-chemistry.org/namedreactions/grignard-reaction.shtm (accessed Mar 28, 2016)

36 Wikipedia. http://en.wikipedia.org/wiki/Grignard_reaction (accessed Mar 28, 2016) 
37 Named Reaction. http://www.organic-chemistry.org/namedreactions/grignard-reaction.shtm (accessed Mar 28, 2016)

38 Wikipedia. http://en.wikipedia.org/wiki/Grignard_reaction (accessed Mar 28, 2016)

39 Named Reaction. http://www.name-reaction.com/negishi-cross-coupling (accessed Mar 28, 2016)

40 Named Reaction. http://www.name-reaction.com/wittig-reaction (accessed Mar 28, 2016)

41 Wikipedia. http://en.wikipedia.org/wiki/Wittig_reaction (accessed Mar 28, 2016)

42 Organic Chemistry Portal. http://www.organic-chemistry.org/namedreactions/friedel-craftsalkylation.shtm (accessed Mar 28, 2016)

43 Scott, R.H.; Barnes, C.; Gerhard, U.; Balasubramanian, S. Exploring a Chemical Encoding Strategy for Combinatorial Synthesis using Friedel-Crafts Alkylation. Chem. Commun., 1999, 14, 1331-2. For Online version, see: http://www.ncbi.nlm.nih.gov/pmc/articles/PMC2231855/ (accessed Mar 28, 2016)

44 Rueping, M.; Nachtsheim, B.J. A Review of New Developments in the Friedel-Crafts Alkylation - From Green Chemistry to Asymmetric Catalysis. Beilstein J. Org. Chem., 2010, 6, 6. For Online version, see: http://www.ncbi.nlm.nih.gov/pmc/articles/PMC2870981/ (accessed Mar 28, 2016)

45 Organic Chemistry Portal. http://www.organic-chemistry.org/namedreactions/friedel-craftsacylation.shtm (accessed Mar 28, 2016)

46 Raja, E.K.; DeSchepper, D.J.; Nilsson Lill, S.O.; Klumpp, D.A. Friedel-Crafts Acylation with Amides. J. Org. Chem., 2012, 77, 5788-93. For Online version, see: http://www.ncbi.nlm.nih.gov/pmc/articles/PMC3402163/ (accessed Mar 28, 2016)

47 Organic Chemistry 4E Carey. http://www.mhhe.com/physsci/chemistry/carey/student/olc/ch12friedelcraftsacylation.html (accessed Mar 28, 2016)

48 Wikipedia. https://en.wikipedia.org/wiki/Michael_reaction (accessed Mar 28, 2016)

49 ThermoFisher Scientific. http://www.piercenet.com/method/amine-reactive-crosslinkerchemistry (accessed Mar 28, 2016)

50 Schurer, S.C.; Tyagi, P.; Muskal, S.M. Prospective Exploration of Synthetically Feasible, Medicinally Relevant Chemical Space. J. Chem. Inf. Model. 2005, 45, 239-248.

51 Wikipedia. http://en.wikipedia.org/wiki/Weinreb_ketone_synthesis (accessed Mar 28, 2016)

52 Kumar, A.; Akula, H.K.; Lakshman, M.K. Simple Synthesis of Amides and Weinreb Amides via Use of $\mathrm{PPh} 3$ or Polymer-Supported PPh3 and Iodine. Eur. J. Org. Chem., 2010, 2010, 10. For Online version, see: http://www.ncbi.nlm.nih.gov/pmc/articles/PMC3818920/ (accessed Mar 28, 2016)

53 Gololobov, Y.G.; Zhmurova, I.N.; Kasukhim, L.F. Sixty Years of Staudinger Reaction. Tetrahedron, 1981, 37, 437-72.

54 Organic Chemistry Portal. http://www.organic-chemistry.org/namedreactions/staudingerreaction.shtm (accessed Mar 28, 2016)

55 Wikipedia. http://en.wikipedia.org/wiki/Beckmann_rearrangement (accessed Mar 28, 2016)

56 Organic Chemistry Portal. http://www.organic-chemistry.org/namedreactions/beckmannrearrangement.shtm (accessed Mar 28, 2016)

57 March, J. Advanced Organic Chemistry: Reactions, Mechanisms, and Structure (3rd ed.), Wiley-interscience publication: New York, 1985.

58 Wikipedia. http://en.wikipedia.org/wiki/Amide_reduction (accessed Mar 28, 2016) 
59 Blackwell, J. M.; Sonmor, E. R.; Scoccitti, T.; Piers, W. E. B(C6F5)3-Catalyzed Hydrosilation of Imines via Silyliminium Intermediates. Org. Lett. 2000, 2, 3921-3923.

60 Organic Chemistry Portal. http://www.organicchemistry.org/synthesis/N1H/reductionsimines.shtm (accessed Mar 28, 2016)

61 Wikipedia. http://en.wikipedia.org/wiki/Nitrile reduction (accessed Mar 28, 2016)

62 Sakai, N.; Moriya, T.; Konakahara, T. An Efficient One-Pot Synthesis of Unsymmetrical Ethers: A Directly Reductive Deoxygenation of Esters Using an InBr3/Et3SiH Catalytic System. J. Org. Chem., 2007, 72, 5920-5922.

63 REDUCTION OF ALDEHYDES AND KETONES. http://www.chemguide.co.uk/organicprops/carbonyls/reduction.html (accessed Mar 28, 2016)

64 Keck, G.E.; Truong, A.P. Directed Reduction of $\beta$-Amino Ketones to Syn or Anti 1,3Amino Alcohol Derivatives. Org. Lett., 2002, 4, 3131-3134. For Online version, see: http://www.ncbi.nlm.nih.gov/pmc/articles/PMC1480404/ (accessed Mar 28, 2016)

65 Organic Chemistry Portal. http://www.organicchemistry.org/synthesis/C1H/reductionsalkenes.shtm (accessed Mar 28, 2016)

66 Cadogan, J.I.G. Reduction of Nitro- and Nitroso-compounds by Tervalent Phosphorus Reagents. Q. Rev. Chem. Soc., 1968, 22, 222-251.

67 Zuman, P.; Shah, B. Addition, Reduction, and Oxidation Reactions of Nitrosobenzene. Chem. Rev., 1994, 94, 1621-41.

68 Kamm, O. $\beta$-Phenylhydroxylamine. Organic Syntheses, 1925, 57-57.

69 Wikipedia. http://en.wikipedia.org/wiki/Reduction_of_nitro_compounds (accessed Mar 28, 2016)

70 Nielsen, A.T. The Isomeric Dinitrocyclohexanes. II. Stereochemistry. J. Org. Chem., 1962, 27, 1998-2001.

71 Wikipedia. http://en.wikipedia.org/wiki/Reduction_of_nitro_compounds (accessed Mar 28, 2016)

72 Hanson, J. R.; Premuzic, E. Applications of Chromous Chloride--II: The Reduction of Some Steroidal Nitro Compounds. Tetrahedron, 1967, 23, 4105-4110.

73 Wikipedia. http://en.wikipedia.org/wiki/Reduction_of_nitro_compounds (accessed Mar 28, 2016)

74 Guttieri, M.J.; Maier, W.F. Selective cleavage of carbon-nitrogen bonds with platinum. J. Org. Chem. 1984, 49, 2875-2880.

75 Wikipedia. http://en.wikipedia.org/wiki/Reduction_of_nitro_compounds (accessed Mar 28, 2016)

76 Wikipedia. http://en.wikipedia.org/wiki/Alcohol_oxidation (accessed Mar 28, 2016)

77 Wikipedia. http://en.wikipedia.org/wiki/Alcohol_oxidation (accessed Mar 28, 2016)

78 Wikipedia. http://en.wikipedia.org/wiki/Alcohol_oxidation (accessed Mar 28, 2016)

79 Bahrami, K.; Khodaei, M.M.; Arabi, M.S. TAPC-Promoted Oxidation of Sulfides and Deoxygenation of Sulfoxides. J. Org. Chem., 2010, 75, 6208-6213.

80 Organic Chemistry Portal. http://www.organic-chemistry.org/synthesis/O2S/sulfoxides.shtm (accessed Mar 28, 2016)

81 Wikipedia. http://en.wikipedia.org/wiki/Sulfone (accessed Mar 28, 2016) 